\title{
COX RINGS OVER NONCLOSED FIELDS
}

\author{
ULRICH DERENTHAL AND MARTA PIEROPAN
}

\begin{abstract}
We give a definition of Cox rings and Cox sheaves for varieties over nonclosed fields that is compatible with torsors under quasitori, including universal torsors. We study their existence and classification, we make the relation to torsors precise, and we present arithmetic applications.
\end{abstract}

\section{Contents}

\author{
1. Introduction \\ 2. Over separably closed fields \\ 3. Over nonclosed fields \\ 4. Finitely generated Cox rings \\ 5. Arithmetic applications \\ References
}

\section{INTRODUCTION}

Starting in the 1970s, Colliot-Thélène and Sansuc studied torsors under quasitori, i.e., under groups of multiplicative type. In particular, they introduced universal torsors of varieties with finitely generated geometric Picard group, which were used to study rational points and other arithmetic questions on geometrically rational varieties over number fields; see CTS87, Sko01.

In the 1990s, Cox Cox95] constructed homogeneous coordinate rings of toric varieties over the field $\mathbb{C}$ of complex numbers. Salberger Sal98] used them in connection with universal torsors to study rational points on split toric varieties over number fields. Homogeneous coordinate rings, or Cox rings, were introduced for more general classes of varieties by Hu, Keel [HK00, and others EKW04, BH03, Hau08. Soon it was observed informally that there should be a close connection to universal torsors (see the introductions of [BP04, HT04, [SS07]). So far, this connection has been made precise only over algebraically closed fields [Has09, Theorem 5.6], ADHL15, §1.6].

The problem of descending Cox rings to nonclosed fields was posed already in BP04, HT04. The purpose of this article is to define Cox rings and Cox sheaves for varieties over fields that are not necessarily algebraically closed in a way that is compatible with the definition of universal torsors and, more generally, of torsors under quasitori.

We start by revisiting and generalizing the definition and construction of Cox rings and Cox sheaves over separably closed fields; see Section 2 Our axiomatic definition allows us to study their existence and classification over arbitrary fields, their functoriality properties, and their precise relation to torsors; see Section 3 . We

Date: September 3, 2018.

2010 Mathematics Subject Classification. 14C20 (11G35, 14L30).

Key words and phrases. Cox ring, Cox sheaf, universal torsor. 
conclude with some properties of finitely generated Cox rings nd their arithmetic applications in Sections 4 and 5] respectively.

It is an active field of research to determine generators and relations of Cox rings over algebraically closed fields for many classes of varieties. Since we also provide explicit methods to derive a description of Cox rings and torsors over nonclosed fields from these, we hope that our work lays the foundation for further arithmetic applications of the vast literature on Cox rings.

1.1. Motivation. Torsors are central tools in the study of rational points on a variety $X$ over a number field $k$. To study the Hasse principle and weak approximation, often a local description of torsors is sufficient; see [CTS87, §3] and [Sko01, Part 2], for example. Such a local description over sufficiently small open subsets $U$ of $X$ can be determined as in [CTS87, §2.3].

A global description of torsors is needed for other applications, such as to Manin's conjecture on the distribution of rational points on Fano varieties [FMT89]. In this application, initiated in Sal98, rational points on a Fano variety $X$ are parameterized by integral points on its universal torsors, and the necessary explicit global description of universal torsors should be provided by Cox rings once their generators and relations are known.

However, the relation between Cox rings and the parameterization of rational points used in proofs of special cases of Manin's conjecture is rarely made precise; of course the lack of a suitable definition of Cox rings over nonclosed fields until now is a fundamental reason for this. In many cases such as [BBD07, a parameterization is obtained by elementary manipulations of the equations defining $X$. If $X$ is split (i.e., the natural map $\operatorname{Pic}(X) \hookrightarrow \operatorname{Pic}\left(X_{\bar{k}}\right)$ from the Picard group over $k$ to the geometric Picard group is an isomorphism), one typically observes that the resulting auxiliary equations are the relations in a Cox ring of $X$, at least over an algebraic closure $\bar{k}$ of $k$, and then one expects that the parameterization is induced by a universal torsor. For nonsplit $X$, the geometric interpretation of the parameterization sometimes remains completely unclear, as in BB07. In other cases such as BBP12, BBS14, (universal) torsors are determined rigorously, while Cox rings remain behind the scenes or are only briefly mentioned.

The only cases where the role of Cox rings is made rigorous seem to be split toric varieties [Sal98] and split weak del Pezzo surfaces [FP16]. For these classes of varieties, the explicit description of Cox rings from [Cox95, HT04, Der14] is successfully applied to prove many cases of Manin's conjecture; see [Sal98, Pie16, FP16, and the references in ADHL15, §6.4.1].

In this article, we develop a theory of Cox rings over nonclosed fields that is suitable to handle torsor parameterizations of rational points for not necessarily split varieties. In order to deal with parameterizations by non-universal torsors, we introduce the notion of Cox rings of a given type, which encode a global description of torsors under quasitori of the same type.

1.2. Cox rings of arbitrary type. In [CTS87, torsors under quasitori are classified in terms of their type. Let $k$ be a field with separable closure $\bar{k}$ and Galois group $\mathfrak{g}=\operatorname{Gal}(\bar{k} / k)$. We recall that there is an antiequivalence of categories between quasitori over $k$ (i.e., smooth groups of multiplicative type of finite type) and finitely generated $\mathfrak{g}$-modules (i.e., $\mathfrak{g}$-modules that are finitely generated as abelian groups) with torsion coprime to the characteristic of $k$, which sends a quasitorus $G$ to its group of characters $M:=\operatorname{Hom}_{\bar{k}}\left(G_{\bar{k}}, \mathbb{G}_{m, \bar{k}}\right)$.

Let $X$ be a variety over $k$ such that $X_{\bar{k}}$ has only constant invertible regular functions. Torsors over $X$ under a quasitorus $G$ with group of characters $M$ are 
classified by the middle term of the exact sequence

$$
0 \rightarrow H_{e ̂ t}^{1}(k, G) \rightarrow H_{e ̂ t}^{1}\left(X, G_{X}\right) \stackrel{\text { type }}{\longrightarrow} \operatorname{Hom}_{\mathfrak{g}}\left(M, \operatorname{Pic}\left(X_{\bar{k}}\right)\right) \rightarrow H_{e ́ t}^{2}(k, G),
$$

from CTS87, Théorème 1.5.1]. The map type sends the isomorphism class of a torsor $Y$ to its type, namely to the homomorphism $\lambda: M \rightarrow \operatorname{Pic}\left(X_{\bar{k}}\right)$ of $\mathfrak{g}$-modules that sends a character $\chi \in M$ to the isomorphism class of the pushforward $\chi_{*} Y$ in $H_{e t}^{1}\left(X_{\bar{k}}, \mathbb{G}_{m}\right)=\operatorname{Pic}\left(X_{\bar{k}}\right)$; see [CTS87, $\left.\S 2.0\right]$. By definition, a universal torsor is a torsor of identity type (i.e., $M=\operatorname{Pic}\left(X_{\bar{k}}\right)$ and $\left.\lambda=\operatorname{id}_{\operatorname{Pic}\left(X_{\bar{k}}\right)}\right)$.

In Definitions 2.1 and 3.3 we introduce the notion of Cox rings of a given type

$$
\lambda: M \rightarrow \operatorname{Pic}\left(X_{\bar{k}}\right),
$$

where $M$ is a finitely generated $\mathfrak{g}$-module whose torsion is coprime to the characteristic of $k$. This is a $k$-algebra $R$ with an $M$-grading on $R_{\bar{k}}$ such that, for every $m \in M$, its degree- $m$-part is isomorphic to $H^{0}(X, \mathcal{O}(D))$ for a divisor $D$ of class $\lambda(m)$; the main difficulty is to define a multiplication. In particular, a Cox ring of identity type is a Cox ring in the original sense.

Below, our results are all stated for Cox rings and Cox sheaves of arbitrary type $\lambda$. The statements for ordinary Cox rings and universal torsors are then recovered by taking the identity type $M:=\operatorname{Pic}\left(X_{\bar{k}}\right)$ and $\lambda:=\operatorname{id}_{\operatorname{Pic}\left(X_{\bar{k}}\right)}$.

1.3. Cox rings over closed fields. In the literature, a Cox ring (of identity type) of a variety $X$ over an algebraically closed field $k$ is a $\operatorname{Pic}(X)$-graded $k$-algebra of the form

$$
R=\bigoplus_{\operatorname{Pic}(X)} H^{0}(X, \mathcal{O}(D))
$$

where $D$ runs through a set of divisors forming a system of representatives of $\operatorname{Pic}(X)$. While the structure of a $\operatorname{Pic}(X)$-graded $k$-vector space is clear, there is no canonical way to define a multiplication on $R$ that turns it into a graded $k$-algebra.

If $X$ is smooth and projective with free Picard group, there is a well-known construction of Cox rings (see HT04, CT06, Has09, TVAV11, for example) that depends on the choice of divisors whose classes form a $\mathbb{Z}$-basis of $\operatorname{Pic}(X)$. It is important to note that a different choice of divisors leads to a Cox ring that is noncanonically isomorphic to the previous one; this makes descending Cox rings an interesting problem (see [BP04, HT04]).

When the Picard group is not free, one can construct Cox rings by choosing divisors whose classes generate $\operatorname{Pic}(X)$ together with a shifting family or an identifying character; see BH03, [ADHL15, §1.4.2]. Another option is to work with the choice of a $k$-rational point on $X$ as in [BP04, Definition 1.1] and [ADHL15, Construction 1.4.2.3].

In our approach to Cox rings over closed fields (see Definition 2.1), two aspects are new: The first novelty is that our definition is axiomatic, while the previously existing definitions can be interpreted as constructions; this allows us to determine automorphisms, to study Galois descent to nonclosed fields, and to phrase and answer existence and uniqueness questions. Our axioms emphasize the role of the divisors associated with every homogeneous element of a Cox ring. The second novelty is that we define Cox rings of arbitrary type.

Then we adapt the previous constructions of Cox rings to the case of arbitrary type (see Construction 2.7) and we show that our axioms are satisfied precisely by the rings obtained from such constructions. In the proof of Theorem 1.2 below, we present several other constructions of Cox rings that seem to be new even over closed fields and are inspired by the theory of torsors.

We recall that one can also construct Cox rings graded by the Weil divisor class group $\mathrm{Cl}(X)$ instead of $\operatorname{Pic}(X)$; see [EKW04, ADHL15]. If $X$ is singular, $\mathrm{Cl}(X)$ may 
differ from Pic $(X)$, and then these Cox rings correspond to good quotients $Y \rightarrow X$ that are not torsors; see ADHL15, §4.2.1]. Hence we only consider Pic $(X)$-graded Cox rings.

Some articles such as HK00, GOST15, CT15 study Cox rings defined via a choice of divisors forming a $\mathbb{Q}$-basis of $\operatorname{Pic}(X) \otimes_{\mathbb{Z}} \mathbb{Q}\left(\right.$ or $\left.\operatorname{Cl}(X) \otimes_{\mathbb{Z}} \mathbb{Q}\right)$. Here, different choices generally lead to nonisomorphic results. This is enough to study finite generation of the resulting rings, but it is clearly not suitable to investigate torsors.

1.4. The definition over nonclosed fields. We define Cox rings of varieties over nonclosed fields by Galois descent. Since Cox rings over separably closed fields may have nontrivial automorphisms (see Proposition 2.17), Galois descent to nonclosed fields is a nontrivial task, if possible at all.

Let $X$ be a variety over a field $k$ with separable closure $\bar{k}$. In Section $\underline{3}$, we define a Cox ring of $X$ of type $\lambda$ to be a $k$-form $R$ of a Cox ring of $X_{\bar{k}}$ of type $\lambda$ such that the action of the Galois group $\mathfrak{g}:=\operatorname{Gal}(\bar{k} / k)$ on $R \otimes_{k} \bar{k}$ is compatible with the Cox ring structure in the sense of our Definition 3.1. This definition, together with the axiomatic definition of Cox rings over separably closed fields, enables us to study the classification and existence of Cox rings over nonclosed fields.

1.5. Classification and existence. Our first main result concerns the relation between Cox rings, Cox sheaves and torsors under quasitori, and yields the classification of Cox rings over nonclosed fields.

Theorem 1.1. Let $k$ be a field. Let $\mathfrak{g}:=\operatorname{Gal}(\bar{k} / k)$, where $\bar{k}$ is a separable closure of $k$. Let $X$ be a k-variety with $\bar{k}\left[X_{\bar{k}}\right]^{\times}=\bar{k}^{\times}$. Let $M$ be a finitely generated $\mathfrak{g}$ module whose torsion is coprime to the characteristic of $k$, and $\lambda: M \rightarrow \operatorname{Pic}\left(X_{\bar{k}}\right)$ a $\mathfrak{g}$-equivariant homomorphism.

The contravariant functor

$$
\begin{aligned}
\{\text { Cox sheaves of } X \text { of type } \lambda\} & \longrightarrow\{X \text {-torsors of type } \lambda\} \\
\mathcal{R} & \longmapsto \operatorname{Spec}_{X} \mathcal{R}
\end{aligned}
$$

is an anti-equivalence of categories.

If the image of $\lambda$ is generated by effective divisor classes, then the covariant functor

$$
\begin{aligned}
\{\text { Cox sheaves of } X \text { of type } \lambda\} & \longrightarrow\{\text { Cox rings of } X \text { of type } \lambda\} \\
\mathcal{R} & \longmapsto \mathcal{R}(X)
\end{aligned}
$$

is an equivalence of categories.

Conversely, a torsor $\pi: Y \rightarrow X$ of type $\lambda$ corresponds to the Cox sheaf $\pi_{*} \mathcal{O}_{Y}$ and to the Cox ring $\mathcal{O}_{Y}(Y)$ of the same type. Given a Cox ring, it is more involved to recover the corresponding Cox sheaf and torsor; see Sections 3 and 4.

Because of the existence of nontrivial automorphisms, a variety $X$ over a nonclosed field $k$ might have no Cox ring (see Example 3.17), or it might have several nonisomorphic Cox rings of the same type, which can be obtained from one such Cox ring by twisting by cocycles.

Our second main result gives existence criteria for Cox rings:

Theorem 1.2. Let $k$ be a field. Let $\mathfrak{g}:=\operatorname{Gal}(\bar{k} / k)$, where $\bar{k}$ is a separable closure of $k$. Let $X$ be a $k$-variety with $\bar{k}\left[X_{\bar{k}}\right]^{\times}=\bar{k}^{\times}$. Let $M$ be a finitely generated $\mathfrak{g}$ module whose torsion is coprime to the characteristic of $k$, and $\lambda: M \rightarrow \operatorname{Pic}\left(X_{\bar{k}}\right)$ a $\mathfrak{g}$-equivariant homomorphism. 
(A) Given two finite $\mathfrak{g}$-invariant sets $\mathcal{M} \subseteq M$ and $\mathcal{D} \subseteq \operatorname{CaDiv}\left(X_{\bar{k}}\right)$ such that $\mathcal{M}$ generates $M$ and $\lambda(M)=\{[D]: D \in \mathcal{D}\}$, we consider the projections

$$
\varphi_{\mathcal{M}}: \bigoplus_{\substack{(m, D) \in \mathcal{M} \times \mathcal{D} \\ \lambda(m)=[D]}} \mathbb{Z}(m, D) \rightarrow M, \quad \varphi_{\mathcal{D}}: \bigoplus_{\substack{(m, D) \in \mathcal{M} \times \mathcal{D} \\ \lambda(m)=[D]}} \mathbb{Z}(m, D) \rightarrow \operatorname{CaDiv}\left(X_{\bar{k}}\right) .
$$

Then Cox sheaves and torsors of type $\lambda$ over $X$ exist if and only if, for one (equivalently, for every) pair $\mathcal{M}, \mathcal{D}$ as above, there exists a $\mathfrak{g}$-equivariant group homomorphism

$$
\chi: \operatorname{ker}\left(\varphi_{\mathcal{M}}\right) \rightarrow \bar{k}(X)^{\times}
$$

such that the principal divisor defined by $\chi(x)$ is $\varphi_{\mathcal{D}}(x)$ for all $x \in \operatorname{ker}\left(\varphi_{\mathcal{M}}\right)$.

(B) For the existence of Cox sheaves, Cox rings and torsors of type $\lambda$ over $X$, the existence of one of the following suffices.

(1) A $\mathfrak{g}$-equivariant splitting of the natural exact sequence of $\mathfrak{g}$-modules

$$
1 \rightarrow \bar{k}^{\times} \rightarrow \bar{k}\left(X_{\bar{k}}\right)^{\times} \rightarrow \bar{k}\left(X_{\bar{k}}\right)^{\times} / \bar{k}^{\times} \rightarrow 1 .
$$

(2) An open subset $U \subseteq X$ such that $\lambda(M)$ is generated by classes of Cartier divisors on $X_{\bar{k}}$ supported outside $U_{\bar{k}}$, with a $\mathfrak{g}$-equivariant splitting of the natural exact sequence of $\mathfrak{g}$-modules

$$
1 \rightarrow \bar{k}^{\times} \rightarrow \bar{k}\left[U_{\bar{k}}\right]^{\times} \rightarrow \bar{k}\left[U_{\bar{k}}\right]^{\times} / \bar{k}^{\times} \rightarrow 1 .
$$

(3) A k-rational point on $X$.

Conversely, if $X_{\bar{k}}$ is locally factorial and $\lambda$ is injective, then the existence of splittings as in (2) for all suitable $U$ is necessary for the existence of Cox sheaves or torsors of $X$ of type $\lambda$. If $k$ is perfect and $X_{\bar{k}}$ is locally factorial, then the existence of a splitting as in (11)) is necessary for the existence of Cox sheaves of type $\operatorname{id}_{\operatorname{Pic}\left(X_{\bar{k}}\right)}$ and universal torsors of $X$.

(C) If $\lambda(M)$ is generated by effective divisor classes, then the existence of Cox sheaves of $X$ of type $\lambda$ is necessary for the existence of Cox rings of $X$ of type $\lambda$.

Part $(A)$ is based on a Galois-equivariant version of Construction 2.7 combined with Galois descent. If $X$ is smooth, then part $(B)$ follows from the existence criteria for torsors from [CTS87, §2.2] via Theorem 1.1]; in particular, the criterion $(B,(1)$ is the vanishing of the elementary obstruction [CTS87, Definition 2.2.1]. In our proof, however, we take the criteria in [CTS87, §2.2] only as guidelines for new Galois-equivariant constructions of Cox rings over $\bar{k}$; then Galois descent gives Cox rings over $k$ (see Constructions 3.20 and 3.23 ). This leads to a proof of the existence criteria $(B)$ for Cox rings that avoids the machinery from CTS87 and also applies to singular varieties; then Theorem 1.1 transfers the existence criteria to torsors in this more general setting.

Note that the existence of a $k$-rational point is not necessary for the existence of Cox rings and Cox sheaves on $X$; see Example 3.25 .

An interpretation of universal torsors in terms of gerbes is given in BV17, §13].

1.6. Applications. In the last sections, we study Cox rings that are finitely generated as $k$-algebras because such a Cox ring $R$ gives a global description of the corresponding torsor as an open subset of the affine variety $\operatorname{Spec} R$. We recall that there is a vast and growing literature computing finitely generated Cox rings of identity type in terms of generators and relations over algebraically closed fields.

In Section 4, we make precise how a Cox ring can be described by generators and relations (note that it is not enough to describe divisors corresponding to the generators, but that one needs a suitable character to obtain a well-defined object). 
We show that a Cox ring $R^{\prime}$ of arbitrary type can be obtained as a pullback of a Cox ring $R$ of identity type, and we explain a method to produce generators and relations of $R^{\prime}$ from those of $R$ if the latter is finitely generated.

In Section [5, we we give examples where the parameterizations appearing in proofs of Manin's conjecture for some nonsplit varieties BB07, BBP12 can be interpreted in terms of Cox rings of type $\operatorname{Pic}(X) \hookrightarrow \operatorname{Pic}\left(X_{\bar{k}}\right)$. Recent work of Destagnol [Des16, Des18] applies our techniques to compute Cox rings of a different injective type to the computation of Peyre's constant in the asymptotic formula predicted by Manin's conjecture for some Châtelet surfaces. In particular, our theory allows to complete the comparison of the leading constant in BT13 with Peyre's constant (see Des18, §5.2]).

For algebraic-geometric applications of our theory, see Dun16] and [LU17].

Acknowledgements. The authors were supported by grants DE 1646/3-1 and ES 60/10-1 of the Deutsche Forschungsgemeinschaft. We thank J.-L. Colliot-Thélène, D. A. Cox, J. Hausen, A. N. Skorobogatov, and the referees for useful remarks and discussions, and we thank S. Keicher for explaining the use of the Maple package HK15. to us. Example 5.3 is based on a discussion with D. Loughran.

\section{Over SEPARABly ClOSED FIELdS}

We start by fixing our setting and some notation. In this section, we work over a separably closed field $k$. Let $X$ be an integral $k$-variety. We always assume that $X$ has only constant invertible regular functions (i.e., $k[X]^{\times}=k^{\times}$, where $k[X]$ denotes the ring of global sections $H^{0}\left(X, \mathcal{O}_{X}\right)$ of the structure sheaf of $\left.X\right)$.

Let $\operatorname{CaDiv}(X)$ be the group of Cartier divisors of $X$. For every divisor $D \in$ $\operatorname{CaDiv}(X)$, we denote by $[D]$ its class in $\operatorname{Pic}(X)$. Given a Cartier divisor $D=$ $\left\{\left(U_{i}, f_{i}\right)\right\}_{i}$, its associated sheaf $\mathcal{O}_{X}(D)$ is the invertible sheaf obtained by gluing $f_{i}^{-1} \mathcal{O}_{U_{i}}$. This is a subsheaf of the constant sheaf associated with the function field $k(X)$ of $X$. Therefore, given two Cartier divisors $D_{1}, D_{2}$ and sections $s_{i} \in$ $H^{0}\left(U, \mathcal{O}_{X}\left(D_{i}\right)\right), i \in\{1,2\}$, the product $s_{1} s_{2} \in H^{0}\left(U, \mathcal{O}_{X}\left(D_{1}+D_{2}\right)\right)$ is well-defined. Given a Cartier divisor $D=\left\{\left(U_{i}, f_{i}\right)\right\}_{i}$ and an element $f \in k(X)^{\times}$, we denote by $\operatorname{div}_{D}(f)$ the Cartier divisor $\left\{\left(U_{i}, f f_{i}\right)\right\}_{i}$. Note that $f \in H^{0}(X, \mathcal{O}(D))$ if and only if $\operatorname{div}_{D}(f)$ is an effective divisor. The support of a Cartier divisor $D=\left\{\left(U_{i}, f_{i}\right)\right\}_{i}$ is

$$
\operatorname{Supp}(D):=\left\{x \in X: f_{i} \notin \mathcal{O}_{X, x}^{\times} \text {if } x \in U_{i}\right\} .
$$

We fix a finitely generated abelian group $M$ whose torsion is coprime to the characteristic of $k$ (i.e., the characteristic of $k$ does not divide the order of its torsion subgroup), and a group homomorphism $\lambda: M \rightarrow \operatorname{Pic}(X)$, which will be the type of our Cox rings, Cox sheaves and torsors. We define

$$
M_{\lambda}:=\{(m, D) \in M \times \operatorname{CaDiv}(X):[D]=\lambda(m)\} .
$$

We denote by $\widehat{M}:=\operatorname{Spec} k[M]$ the quasitorus dual to $M$ under the antiequivalence of categories recalled in Section 1.2

Definition 2.1. A Cox ring of $X$ of type $\lambda$ is an $M$-graded $k$-algebra $R$ together with a map

$$
\operatorname{div}: \bigcup_{m \in M}\left(R_{m} \backslash\{0\}\right) \rightarrow \operatorname{CaDiv}(X),
$$

where $R_{m}$ denotes the degree-m-part of $R$, such that, for each $(m, D) \in M_{\lambda}$, there exists a $k$-vector space isomorphism $\phi_{m, D}: R_{m} \rightarrow H^{0}\left(X, \mathcal{O}_{X}(D)\right)$ satisfying $\operatorname{div}(s)=\operatorname{div}_{D}\left(\phi_{m, D}(s)\right)$ for all nonzero $s \in R_{m}$, and $\operatorname{div}\left(s_{1} s_{2}\right)=\operatorname{div}\left(s_{1}\right)+\operatorname{div}\left(s_{2}\right)$ for all nonzero homogeneous elements $s_{1}, s_{2}$ of $R$.

Let $R$ and $R^{\prime}$ be two Cox rings of $X$ of type $\lambda$. A morphism $\psi: R \rightarrow R^{\prime}$ of $M$ graded $k$-algebras is a morphism of Cox rings of $X$ of type $\lambda$ if $\operatorname{div}^{\prime}(\psi(s))=\operatorname{div}(s)$ 
for all nonzero homogeneous $s \in R$, where div and $\operatorname{div}^{\prime}$ are the maps associated with $R$ and $R^{\prime}$, respectively.

A structure of Cox ring of $X$ of type $\lambda$ on a $k$-algebra $R$ consists of an $M$-grading on $R$ and a map div as above.

Note that we only require the existence of the isomorphisms $\phi_{m, D}$; they are not part of the data. We will see in Remark 2.5 that they are determined uniquely up to nonzero constants by the map div if they exist.

In BH03, ADHL15, a Cox ring is the ring of global sections of a Cox sheaf. Now we define Cox sheaves of type $\lambda$. In Proposition 2.11, we prove that the Cox rings in Definition 2.1 are the rings of global sections of the Cox sheaves defined below.

Definition 2.2. A Cox sheaf of $X$ of type $\lambda$ is a sheaf $\mathcal{R}$ of $M$-graded $\mathcal{O}_{X}$-algebras together with a family of isomorphisms of $\mathcal{O}_{X}$-modules

$$
\left\{\phi_{m, D}: \mathcal{R}_{m} \rightarrow \mathcal{O}_{X}(D)\right\}_{(m, D) \in M_{\lambda}},
$$

where $\mathcal{R}_{m}$ denotes the degree- $m$-part of $\mathcal{R}$, such that, for every $\left(m_{1}, D_{1}\right),\left(m_{2}, D_{2}\right) \in$ $M_{\lambda}$, there exists a nonzero constant $\alpha \in k$ that satisfies

$$
\phi_{m_{1}, D_{1}}\left(s_{1}\right) \phi_{m_{2}, D_{2}}\left(s_{2}\right)=\alpha \phi_{m_{1}+m_{2}, D_{1}+D_{2}}\left(s_{1} s_{2}\right)
$$

for all $s_{i} \in \mathcal{R}_{m_{i}}(U), i \in\{1,2\}$, and all open subsets $U$ of $X$.

A morphism of Cox sheaves of $X$ of type $\lambda$ is a morphism of $M$-graded $\mathcal{O}_{X^{-}}$ algebras.

A structure of Cox sheaf of $X$ of type $\lambda$ on a sheaf $\mathcal{R}$ of $\mathcal{O}_{X}$-algebras consists of an $M$-grading on $\mathcal{R}$ and a family of isomorphisms $\left\{\phi_{m, D}\right\}_{(m, D) \in M_{\lambda}}$ as above.

Proposition 2.3. An $M$-graded k-algebra $R$ has a structure of Cox ring of $X$ of type $\lambda$ if and only if there exists a family of isomorphisms of $k$-vector spaces

$$
\left\{\phi_{m, D}: R_{m} \rightarrow H^{0}\left(X, \mathcal{O}_{X}(D)\right)\right\}_{(m, D) \in M_{\lambda}},
$$

such that, for every $\left(m_{1}, D_{1}\right),\left(m_{2}, D_{2}\right) \in M_{\lambda}$, there exists a nonzero constant $\alpha \in k$ that satisfies

$$
\phi_{m_{1}, D_{1}}\left(s_{1}\right) \phi_{m_{2}, D_{2}}\left(s_{2}\right)=\alpha \phi_{m_{1}+m_{2}, D_{1}+D_{2}}\left(s_{1} s_{2}\right)
$$

for all $s_{i} \in R_{m_{i}}, i \in\{1,2\}$.

In particular, if $\mathcal{R}$ is a Cox sheaf of $X$ of type $\lambda$, then $\mathcal{R}(X)$ is a Cox ring of $X$ of type $\lambda$.

Proof. If $R$ is an $M$-graded $k$-algebra endowed with a family of isomorphisms $\phi_{m, D}$ as in the statement, for every $m \in M$, choose a divisor $D \in \operatorname{CaDiv}(X)$ such that $(m, D) \in M_{\lambda}$ and define $\operatorname{div}(s):=\operatorname{div}_{D}\left(\phi_{m, D}(s)\right)$ for all nonzero $s \in R_{m}$.

For the converse implication, assume that $R$ is a Cox ring of $X$ of type $\lambda$ with a map div. We choose isomorphisms $\phi_{m, D}$ as in Definition 2.1. We prove that the family of isomorphisms $\phi_{m, D}$ defines on $R$ a structure of Cox ring of $X$ of type $\lambda$ as in the statement. Let $\left(m_{1}, D_{1}\right),\left(m_{2}, D_{2}\right) \in M_{\lambda}$. If $R_{m_{1}}=0$ or $R_{m_{2}}=0$, let $\alpha:=1$. If $s_{i} \in R_{m_{i}} \backslash\{0\}, i \in\{1,2\}$, the elements

$$
\alpha:=\phi_{m_{1}, D_{1}}\left(s_{1}\right) \phi_{m_{2}, D_{2}}\left(s_{2}\right) \phi_{m_{1}+m_{2}, D_{1}+D_{2}}\left(s_{1} s_{2}\right)^{-1}
$$

belong to $k^{\times}$because

$$
\operatorname{div}_{D_{1}+D_{2}}\left(\phi_{m_{1}+m_{2}, D_{1}+D_{2}}\left(s_{1} s_{2}\right)\right)=\operatorname{div}_{D_{1}}\left(\phi_{m_{1}, D_{1}}\left(s_{1}\right)\right)+\operatorname{div}_{D_{2}}\left(\phi_{m_{2}, D_{2}}\left(s_{2}\right)\right),
$$

and do not depend on the chosen sections $s_{i} \in R_{m_{i}} \backslash\{0\}$ because the morphisms $\phi_{m, D}$ are linear. 
We observe that a structure of Cox ring or of Cox sheaf is actually determined by a subfamily of isomorphisms $\phi_{m, D}$. This is made precise via the following lemma, which characterizes the morphisms of $\mathcal{O}_{X}$-modules between invertible sheaves.

Lemma 2.4. Let $D, D^{\prime}$ be two Cartier divisors and $\psi: \mathcal{O}_{X}(D) \rightarrow \mathcal{O}_{X}\left(D^{\prime}\right)$ a morphism of $\mathcal{O}_{X}$-modules. Then there exists an $f \in k(X)$ such that $\psi(s)=f s$ for all $s \in H^{0}\left(U, \mathcal{O}_{X}(D)\right)$ and all open subsets $U \subseteq X$. If $D=D^{\prime}$, then $f \in k[X]$. If $\psi$ is an isomorphism, then $D=\operatorname{div}_{D^{\prime}}(f)$.

Proof. Without loss of generality, we can assume that $D$ and $D^{\prime}$ are trivialized by the same open covering of $X$, say $D=\left\{\left(U_{i}, f_{i}\right)\right\}_{i}$ and $D^{\prime}=\left\{\left(U_{i}, f_{i}^{\prime}\right)\right\}_{i}$. Let $s \in H^{0}\left(U_{i}, \mathcal{O}_{X}(D)\right)$. Then $\psi(s)=\psi\left(f_{i}^{-1}\right) f_{i} s$, and $\psi\left(f_{i}^{-1}\right)=u_{i} f_{i}^{\prime-1}$ for some $u_{i} \in H^{0}\left(U_{i}, \mathcal{O}_{X}\right)$. Moreover, $u_{i} f_{i}^{\prime-1} f_{i}=u_{j} f_{j}^{\prime-1} f_{j}$ in $k(X)$ for all $i$ and $j$ by restricting $\psi$ to $U_{i} \cap U_{j}$. Take $f=u_{i} f_{i}^{\prime-1} f_{i}$.

If $D=D^{\prime}$, then $f \in H^{0}\left(U_{i}, \mathcal{O}_{X}\right)$ for all $i$, hence $f \in k[X]$. If $\psi$ is surjective, then $u_{i} \in H^{0}\left(U_{i}, \mathcal{O}_{X}\right)^{\times}$is a unit for all $i$.

Remark 2.5. The isomorphisms $\phi_{m, D}$ in Definition 2.2 and Proposition 2.3 are uniquely determined up to multiplication by nonzero elements of $k$. Therefore, given a Cox sheaf $\mathcal{R}$ of $X$ of type $\lambda$, we say that two families $\left\{\phi_{m, D}\right\}_{(m, D) \in M_{\lambda}}$ and $\left\{\phi_{m, D}^{\prime}\right\}_{(m, D) \in M_{\lambda}}$ define the same Cox sheaf structure on $\mathcal{R}$ if, for every $(m, D) \in$ $M_{\lambda}$, there is $\alpha_{m, D} \in k^{\times}$such that $\phi_{m, D}^{\prime}=\alpha_{m, D} \phi_{m, D}$.

By Lemma 2.4, a Cox sheaf structure on $\mathcal{R}$ is determined once, for each $m \in M$, the isomorphism $\phi_{m, D}$ is known for one $(m, D) \in M_{\lambda}$.

Analogous remarks apply to Cox rings.

Remark 2.6. Using the equivalent characterization of Proposition 2.3, a morphism of $M$-graded $k$-algebras $\psi: R \rightarrow R^{\prime}$ between two Cox rings of $X$ of type $\lambda$ is a morphism of Cox rings of $X$ of type $\lambda$ as in Definition 2.1 if and only if it is compatible with the families of isomorphisms $\left\{\phi_{m, D}\right\}_{(m, D) \in M_{\lambda}}$ and $\left\{\phi_{m, D}^{\prime}\right\}_{(m, D) \in M_{\lambda}}$ associated with $R$ and $R^{\prime}$, respectively, as follows: for every $(m, D) \in M_{\lambda}$, there is $\alpha_{m, D} \in k^{\times}$such that $\phi_{m, D}^{\prime} \circ \psi \circ \phi_{m, D}^{-1}=\alpha_{m, D} \operatorname{id}_{H^{0}\left(X, \mathcal{O}_{X}(D)\right)}$. Moreover, a structure of Cox ring of $X$ of type $\lambda$ on a $k$-algebra $R$ is an $M$-grading on $R$ and a family of isomorphisms $\left\{\phi_{m, D}\right\}_{(m, D) \in M_{\lambda}}$ as in Proposition 2.3.

Our axiomatic definition of Cox sheaves is compatible with the previous definitions of Pic $(X)$-graded Cox sheaves as follows. The sheaves defined in BH03, Lemma 3.5], with the family of isomorphisms in [BH03, Lemma 3.5(ii)], are Cox sheaves of identity type in the sense of Definition 2.2. If $X$ is locally factorial, the Cox sheaves constructed in ADHL15, Construction 1.4.2.1], with the family of isomorphisms in ADHL15, Lemma 1.4.3.4], are Cox sheaves of identity type in the sense of Definition 2.2 .

The following construction is analogous to [BH03, §3], Hau08, §2], and [ADHL15, Construction 1.4.2.1]. In Proposition 2.9, we will show that every Cox sheaf of $X$ of type $\lambda$ is isomorphic to one defined in Construction 2.7 and that the Cox sheaves obtained from Construction 2.7 are all isomorphic.

Construction 2.7. Let

$$
0 \rightarrow \Lambda_{0} \rightarrow \Lambda \stackrel{\varphi}{\rightarrow} M \rightarrow 0
$$

be a presentation of $M$ by a finitely generated free group $\Lambda$. Let $L_{1}, \ldots, L_{n}$ be a basis of $\Lambda$. For $i \in\{1, \ldots, n\}$, let $D_{i}$ be a Cartier divisor representing the class $\lambda\left(\varphi\left(L_{i}\right)\right)$ in $\operatorname{Pic}(X)$. For every $L=\sum_{i=1}^{n} a_{i} L_{i}$ in $\Lambda$, let $\mathcal{S}_{L}:=\mathcal{O}_{X}\left(\sum_{i=1}^{n} a_{i} D_{i}\right)$. Endow $\mathcal{S}:=\bigoplus_{L \in \Lambda} \mathcal{S}_{L}$ with the multiplication of sections induced by the embeddings $\mathcal{S}_{L} \subseteq k(X)$. Let $\chi: \Lambda_{0} \rightarrow k(X)^{\times}$be a homomorphism of groups that satisfies $\operatorname{div}_{0}(\chi(E))=\sum_{i=1}^{n} a_{i} D_{i}$ for all $E=\sum_{i=1}^{n} a_{i} L_{i} \in \Lambda_{0}$. We call $\chi$ a character 
associated with $\mathcal{S}$. Let $\mathcal{I}$ be the sheaf of ideals of $\mathcal{S}$ locally generated by the sections $1-\chi(E)$ for all $E \in \Lambda_{0}$, where 1 has degree 0 and $\chi(E)$ has degree $-E$. We say that $\mathcal{I}$ is the sheaf of ideals of $\mathcal{S}$ defined by $\chi$. Let $\mathcal{R}:=\mathcal{S} / \mathcal{I}$ and $\pi: \mathcal{S} \rightarrow \mathcal{R}$ the projection. Then

(1) $\mathcal{I}$ is $M$-homogeneous, and every $\Lambda$-homogeneous section of $\mathcal{I}$ is zero;

(2) $\left.\pi\right|_{\mathcal{S}_{L}}: \mathcal{S}_{L} \rightarrow \mathcal{R}_{\varphi(L)}$ is an isomorphism of $\mathcal{O}_{X}$-modules for all $L \in \Lambda$;

(3) $\mathcal{R}$ with a family of isomorphisms $\left\{\phi_{m, D}\right\}_{(m, D) \in M_{\lambda}}$ induced by $\left\{\left.\pi\right|_{\mathcal{S}_{L}} ^{-1}\right\}_{L \in \Lambda}$ is a Cox sheaf of $X$ of type $\lambda$.

Proof. Since $\varphi(E)=0$ for all $E \in \Lambda_{0}$, the sheaf $\mathcal{I}$ is $M$-homogeneous and $\mathcal{R}$ is $M$ graded. To prove (1) let $U$ be an open subset of $X$ and $s \in H^{0}(U, \mathcal{I})$ a homogeneous section of degree $L \in \Lambda$. Let $\mathcal{V}$ be an open covering of $U$ such that, for all $V \in \mathcal{V}$, we can write $\left.s\right|_{V}=\sum_{E \in \Lambda_{0}}(1-\chi(E)) s_{E}$ with $s_{E} \in H^{0}\left(V, \bigoplus_{E^{\prime} \in \Lambda_{0}} \mathcal{S}_{L+E^{\prime}}\right)$ for all $E \in \Lambda_{0}$. Write $s_{E}=\sum_{E^{\prime} \in \Lambda_{0}} s_{E, E^{\prime}}$ with $s_{E, E^{\prime}} \in H^{0}\left(V, \mathcal{S}_{L+E^{\prime}}\right)$. Then

$$
\left.s\right|_{V}=\sum_{E, E^{\prime} \in \Lambda_{0}}\left(\left(1-\chi\left(E-E^{\prime}\right)\right) \chi\left(E^{\prime}\right) s_{E, E^{\prime}}-\left(1-\chi\left(-E^{\prime}\right)\right) \chi\left(E^{\prime}\right) s_{E, E^{\prime}}\right),
$$

where $\chi\left(E^{\prime}\right) s_{E, E^{\prime}}$ is homogeneous of degree $L$. Hence $\left.s\right|_{V}=\sum_{E \in \Lambda_{0}}(1-\chi(E)) s_{E}^{\prime}$ for suitable $s_{E}^{\prime} \in H^{0}\left(V, \mathcal{S}_{L}\right)$. Since $s$ is homogeneous of degree $L$, we get $\left.s\right|_{V}=0$ for all $V \in \mathcal{V}$. Hence $s=0$.

For (2), note that $\left.\pi\right|_{\mathcal{S}_{L}}$ is injective for all $L \in \Lambda$ by (1), Let $U$ be an open subset of $X$ and $L \in \Lambda$. We prove that $\left.\pi\right|_{H^{0}\left(U, \mathcal{S}_{L}\right)}: H^{0}\left(U, \mathcal{S}_{L}\right) \rightarrow H^{0}\left(U, \mathcal{R}_{\varphi(L)}\right)$ is surjective. Let $s \in H^{0}\left(U, \mathcal{R}_{\varphi(L)}\right)$ and $\mathcal{V}$ an open covering of $U$ such that for all $V \in \mathcal{V}$ there exists a section $s_{V} \in H^{0}\left(V, \bigoplus_{E \in \Lambda_{0}} \mathcal{S}_{L+E}\right)$ with $\pi\left(s_{V}\right)=\left.s\right|_{V}$. Write $s_{V}=\sum_{E \in \Lambda_{0}} s_{V, E}$ with $s_{V, E}$ homogeneous of degree $L+E$. Then $s_{V}^{\prime}:=$ $s_{V}-\sum_{E \in \Lambda_{0}}(1-\chi(E)) s_{V, E}$ is homogeneous of degree $L$ and $\pi\left(s_{V}^{\prime}\right)=\left.s\right|_{V}$. Since $\left.s_{V}^{\prime}\right|_{V \cap W}-\left.s_{W}^{\prime}\right|_{V \cap W}$ is a $\Lambda$-homogeneous section of $\mathcal{I}$, the sections $s_{V}^{\prime}$ glue by (1) to a section $s^{\prime} \in H^{0}\left(U, \mathcal{S}_{L}\right)$ such that $\pi\left(s^{\prime}\right)=s$.

Finally, we prove (3). For every $(m, D) \in M_{\lambda}$, choose $L \in \varphi^{-1}(m)$ and an isomorphism of $\mathcal{O}_{X}$-modules $\psi_{L, D}: \mathcal{S}_{L} \rightarrow \mathcal{O}_{X}(D)$. Then

$$
\left\{\phi_{m, D}:=\left.\psi_{L, D} \circ \pi\right|_{\mathcal{S}_{L}} ^{-1}: \mathcal{R}_{m} \rightarrow \mathcal{O}_{X}(D)\right\}_{(m, D) \in M_{\lambda}}
$$

is a family of isomorphisms induced by $\left\{\left.\pi\right|_{\mathcal{S}_{L}} ^{-1}\right\}_{L \in \Lambda}$. By Lemma 2.4, for every $\left(m_{1}, D_{1}\right),\left(m_{2}, D_{2}\right) \in M_{\lambda}$, there exists a nonzero constant $\alpha \in k$ that satisfies

$$
\phi_{m_{1}, D_{1}}\left(s_{1}\right) \phi_{m_{2}, D_{2}}\left(s_{2}\right)=\alpha \phi_{m_{1}+m_{2}, D_{1}+D_{2}}\left(s_{1} s_{2}\right)
$$

for all $s_{i} \in H^{0}\left(U, \mathcal{R}_{m_{i}}\right), i \in\{1,2\}$, and all open subsets $U$ of $X$.

Since $\Lambda$ is free and finitely generated, the same is true for $\Lambda_{0}$. Therefore, characters $\chi$ as in Construction 2.7 always exist.

Proposition 2.8. The Cox sheaves defined in Construction 2.7 do not depend on the choice of the character $\chi$, up to isomorphism of Cox sheaves.

Proof. Let $\Lambda, \Lambda_{0}$, and $\mathcal{S}$ be as in Construction 2.7. Let $\chi$ and $\chi^{\prime}$ be two characters associated with $\mathcal{S}$, and $\mathcal{I}$ and $\mathcal{I}^{\prime}$ the sheaves of ideals of $\mathcal{S}$ defined by $\chi$ and $\chi^{\prime}$, respectively. Since $\Lambda$ is free and finitely generated, $k$ is separably closed and the order of the torsion subgroup of $M$ is not divisible by the characteristic of $k$, the character $\chi^{\prime} \chi^{-1}: \Lambda_{0} \rightarrow k^{\times}$extends to a character $\alpha: \Lambda \rightarrow k^{\times}=k[X]^{\times}$, which defines an automorphism $\psi: \mathcal{S} \rightarrow \mathcal{S}$ by sending each homogeneous element $s$ of degree $L$ to $\alpha(-L) s$. Since $\psi(1-\chi(E))=1-\alpha(E) \chi(E)=1-\chi^{\prime}(E)$ for all $E \in \Lambda_{0}$, the automorphism $\psi$ maps $\mathcal{I}$ onto $\mathcal{I}^{\prime}$. Hence it induces an isomorphism $\mathcal{S} / \mathcal{I} \rightarrow \mathcal{S} / \mathcal{I}^{\prime}$ of $M$-graded $\mathcal{O}_{X}$-algebras. 
Proposition 2.9. Let $\mathcal{R}$ be a Cox sheaf of $X$ of type $\lambda$. For every $\mathcal{S}$ as in Construction 2.7. there exists a character $\chi$ associated with $\mathcal{S}$ and an isomorphism $\mathcal{R} \cong \mathcal{S} / \mathcal{I}$ of Cox sheaves of type $\lambda$, where $\mathcal{I}$ is the sheaf of ideals of $\mathcal{S}$ defined by $\chi$.

Proof. Let $\Lambda_{0}, \Lambda, \varphi$ and $\mathcal{S}$ be as in Construction 2.7. Let $\mathcal{S}^{\prime}:=\bigoplus_{L \in \Lambda} \mathcal{R}_{\varphi(L)}$. Let $\phi_{m, D}: \mathcal{R}_{m} \rightarrow \mathcal{O}_{X}(D)$, for $(m, D) \in M_{\lambda}$, be a family of isomorphisms associated with $\mathcal{R}$.

Let $\mathcal{B}$ be the basis of $\Lambda$ chosen in Construction 2.7 and let $\Lambda_{+}$be the monoid generated by $\mathcal{B}$. For every $L \in \mathcal{B}$, denote by $D_{L}$ the Cartier divisor representing the class $\lambda(\varphi(L))$ in $\operatorname{Pic}(X)$ such that $\mathcal{S}_{L}=\mathcal{O}_{X}\left(D_{L}\right)$. For every $L \in \Lambda_{+}$, write $L=\sum_{i=1}^{r} L_{i}$ with not necessarily distinct $L_{i} \in \mathcal{B}$, define $D_{L}:=\sum_{i=1}^{r} D_{L_{i}}$, and let $\alpha_{L}$ be the unique element of $k^{\times}$that satisfies

$$
\prod_{i=1}^{r} \phi_{\varphi\left(L_{i}\right), D_{L_{i}}}\left(s_{i}\right)=\alpha_{L} \phi_{\varphi(L), D_{L}}\left(s_{1} \cdots s_{r}\right)
$$

for all $s_{i} \in H^{0}\left(U, \mathcal{R}_{\varphi\left(L_{i}\right)}\right)$ and all open $U \subseteq X$. For every $L \in \Lambda$, write $L=$ $L^{+}-L^{-}$with $L^{+}, L^{-} \in \Lambda_{+}$, and define $\alpha_{L}:=\alpha_{L^{+}} \alpha_{L^{-}}^{-1} \alpha^{-1}$, where $\alpha \in k^{\times}$is the unique constant that satisfies $\phi_{\varphi(L), D_{L}}(s) \phi_{\varphi\left(L^{-}\right), D_{L^{-}}}\left(s^{\prime}\right)=\alpha \phi_{\varphi\left(L^{+}\right), D_{L^{+}}}\left(s s^{\prime}\right)$ for all $s \in H^{0}\left(U, \mathcal{R}_{\varphi(L)}\right), s^{\prime} \in H^{0}\left(U, \mathcal{R}_{\varphi\left(L^{-}\right)}\right)$and all open $U \subseteq X$. The constant $\alpha_{L}$ does not depend on the choice of $L^{+}$and $L^{-}$. The morphisms $\psi_{L}:=\alpha_{L} \phi_{\varphi(L), D_{L}}$ induce an isomorphism of $\Lambda$-graded $\mathcal{O}_{X}$-algebras $\psi: \mathcal{S}^{\prime} \rightarrow \mathcal{S}$.

The map $\chi: \Lambda_{0} \rightarrow k(X)^{\times}$defined by $\chi(E):=\psi_{-E}(1)$ for all $E \in \Lambda_{0}$, is a character associated with $\mathcal{S}$ by Lemma 2.4. Let $\mathcal{I}^{\prime}$ be the kernel of the projection $\mathcal{S}^{\prime} \rightarrow \mathcal{R}$. Then $\psi\left(\mathcal{I}^{\prime}\right)$ is the sheaf $\mathcal{I}$ of ideals of $\mathcal{S}$ defined by $\chi$. Thus $\psi$ induces an isomorphism $\mathcal{R} \cong \mathcal{S} / \mathcal{I}$.

Corollary 2.10. There exists exactly one isomorphism class of Cox sheaves of $X$ of type $\lambda$.

Proposition 2.11. Every Cox ring of $X$ of type $\lambda$ is isomorphic, as a Cox ring of type $\lambda$, to the ring of global sections of a Cox sheaf of $X$ of type $\lambda$.

Proof. Let $R$ be a Cox ring of $X$ of type $\lambda$. Let $\phi_{m, D}: R_{m} \rightarrow H^{0}\left(X, \mathcal{O}_{X}(D)\right.$ ), for $(m, D) \in M_{\lambda}$, be a family of isomorphisms associated with $R$ as in Proposition 2.3.

Let $\Lambda_{0}, \Lambda, \varphi$ and $\mathcal{S}$ be as in Construction 2.7. For every $L \in \Lambda$, let $D_{L}$ be the Cartier divisor on $X$ such that $\mathcal{S}_{L}=\mathcal{O}_{X}\left(D_{L}\right)$. Let $\Lambda_{\text {eff }}$ be the subgroup of $\Lambda$ generated by the elements $L$ such that $\lambda(\varphi(L))$ is effective in $\operatorname{Pic}(X)$. We observe that $\Lambda_{0} \subseteq \Lambda_{\text {eff }}$. Since $\Lambda$ is free and finitely generated, the same is true for $\Lambda_{\text {eff }}$. Let $\mathcal{B}_{\text {eff }}$ be a basis of $\Lambda_{\text {eff }}$, and let $\Lambda_{+} \subseteq \Lambda_{\text {eff }}$ be the monoid generated by $\mathcal{B}_{\text {eff }}$. For every $L \in \Lambda_{+}$, write $L=\sum_{i=1}^{r} L_{i}$ with not necessarily distinct $L_{i} \in \mathcal{B}_{\text {eff }}$, and let $\alpha_{L}$ be the unique element of $k^{\times}$that satisfies

$$
\prod_{i=1}^{r} \phi_{\varphi\left(L_{i}\right), D_{L_{i}}}\left(s_{i}\right)=\alpha_{L} \phi_{\varphi(L), D_{L}}\left(s_{1} \cdots s_{r}\right)
$$

for all $s_{i} \in R_{\varphi\left(L_{i}\right)}$ with $i \in\{1, \ldots, r\}$. For every $L \in \Lambda_{\text {eff }}$ such that $\lambda(\varphi(L))$ is effective in $\operatorname{Pic}(X)$, write $L=L^{+}-L^{-}$with $L^{+}, L^{-} \in \Lambda_{+}$, and define $\alpha_{L}:=$ $\alpha_{L^{+}} \alpha_{L^{-}}^{-1} \alpha^{-1}$, where $\alpha \in k^{\times}$is the unique constant that satisfies

$$
\phi_{\varphi(L), D_{L}}(s) \phi_{\varphi\left(L^{-}\right), D_{L^{-}}}\left(s^{\prime}\right)=\alpha \phi_{\varphi\left(L^{+}\right), D_{L^{+}}}\left(s s^{\prime}\right)
$$

for all $s \in R_{\varphi(L)}$ and $s^{\prime} \in R_{\varphi\left(L^{-}\right)}$. The constant $\alpha_{L}$ does not depend on the choice of $L^{+}$and $L^{-}$. The isomorphisms $\psi_{L}:=\alpha_{L}^{-1} \phi_{\varphi(L), D_{L}}^{-1}$ induce a surjective morphism of graded $k$-algebras $\psi: H^{0}(X, \mathcal{S}) \rightarrow R$.

The map $\chi: \Lambda_{0} \rightarrow k(X)^{\times}$defined by $\chi(E):=\psi_{-E}^{-1}(1)$ for all $E \in \Lambda_{0}$ is a character associated with $\mathcal{S}$. Let $\mathcal{I}$ be the sheaf of ideals of $\mathcal{S}$ associated with $\chi$. 
Then $\mathcal{R}:=\mathcal{S} / \mathcal{I}$ is a Cox sheaf of $X$ of type $\lambda, H^{0}(X, \mathcal{S}) / H^{0}(X, \mathcal{I}) \cong H^{0}(X, \mathcal{R})$ by Construction 2.7](2), and $H^{0}(X, \mathcal{I})$ is the kernel of $\psi$.

We show that the induced isomorphism $\psi: H^{0}(X, \mathcal{S}) / H^{0}(X, \mathcal{I}) \rightarrow R$ is a morphism of Cox rings. The Cox sheaf $\mathcal{R}$ is endowed with the family of isomorphisms $\left\{\phi_{m, D}^{\prime}\right\}_{(m, D) \in M_{\lambda}}$ induced by $\left\{\left.\pi\right|_{\mathcal{S}_{L}} ^{-1}\right\}_{L \in \Lambda}$, where $\pi: \mathcal{S} \rightarrow \mathcal{R}$ is the projection. For every $m \in M$ such that $\lambda(m)$ is effective, let $L \in \Lambda_{\text {eff }}$ be such that $\varphi(L)=m$. Without loss of generality, we can assume that $\phi_{m, D_{L}}^{\prime}=\left.\pi\right|_{\mathcal{S}_{L}} ^{-1}$. Hence

$$
\phi_{m, D_{L}} \circ \psi \circ\left(\phi_{m, D_{L}}^{\prime}\right)^{-1}=\alpha_{L}^{-1} \operatorname{id}_{H^{0}\left(X, \mathcal{O}_{X}\left(D_{L}\right)\right)} .
$$

By definition, the degree-m-part of a Cox ring of $X$ of type $\lambda$ is nonzero if and only if $\lambda(m)$ is an effective class in $\operatorname{Pic}(X)$. But the degree- $m$-part of a Cox sheaf of $X$ of type $\lambda$ is always nonzero. Hence we may expect that a Cox sheaf is not completely determined by its ring of global sections. The following example shows that Cox sheaves of different types, which are not isomorphic, may have rings of global sections that are isomorphic as $k$-algebras.

Example 2.12. A Cox ring of $\mathbb{P}^{1} \times \mathbb{P}^{1}$ of identity type is the $\mathbb{Z}^{2}$-graded ring

$$
k\left[x_{0}, x_{1}, y_{0}, y_{1}\right]=\bigoplus_{(a, b) \in \mathbb{Z}_{\geq 0} \times \mathbb{Z}_{\geq 0}} k\left[x_{0}, x_{1}, y_{0}, y_{1}\right]_{(a, b)},
$$

where $x_{0}, x_{1}$ have degree $(1,0)$ and $y_{0}, y_{1}$ have degree $(0,1)$, via the standard identification $\operatorname{Pic}\left(\mathbb{P}^{1} \times \mathbb{P}^{1}\right) \cong \mathbb{Z}^{2}$.

Let $\lambda: \mathbb{Z} \rightarrow \mathbb{Z}^{2}$ be the morphism that sends $a$ to $(a,-a)$. A Cox ring of $\mathbb{P}^{1} \times \mathbb{P}^{1}$ of type $\lambda$ is, by pullback (see Definition 3.12),

$$
\bigoplus_{a \in \mathbb{Z}} k\left[x_{0}, x_{1}, y_{0}, y_{1}\right]_{(a,-a)}=k,
$$

which is the same, as a $k$-algebra, as a Cox ring of $\mathbb{P}^{1} \times \mathbb{P}^{1}$ of type $\{0\} \rightarrow \mathbb{Z}^{2}$. A Cox sheaf of type $\lambda$ is isomorphic to $\bigoplus_{a \in \mathbb{Z}} \mathscr{L}^{\otimes a}$, where $\mathscr{L}$ is an invertible sheaf of type $(1,-1)$, while a Cox sheaf of type $\{0\} \rightarrow \mathbb{Z}^{2}$ is $\mathcal{O}_{\mathbb{P}^{1} \times \mathbb{P}^{1}}$.

Remark 2.13. The example above can be explained as follows. Let $M_{\text {eff }}$ denote the subgroup of $M$ generated by the elements $m$ such that $\lambda(m)$ is effective in $\operatorname{Pic}(X)$, and define $\lambda_{\text {eff }}:=\left.\lambda\right|_{M_{\text {eff }}}: M_{\text {eff }} \rightarrow \operatorname{Pic}(X)$. A Cox ring of $X$ of type $\lambda$ also has a structure of Cox ring of $X$ of type $\lambda^{\prime}$, for all $\lambda^{\prime}: M^{\prime} \rightarrow \operatorname{Pic}(X)$ such that $M_{\text {eff }}^{\prime}=M_{\text {eff }}$ and $\lambda_{\text {eff }}^{\prime}=\lambda_{\text {eff }}$. The situation in the example above cannot occur if $\lambda(M)$ is generated by effective divisor classes. This is the case, for example, if $\lambda(M)$ contains an ample divisor class.

We recall that the morphisms of Cox sheaves of type $\lambda$ are by definition just morphisms of graded $\mathcal{O}_{X}$-algebras, while the morphisms of Cox rings are morphisms of graded $k$-algebras that are compatible with the map div. The following example shows that a $k$-algebra morphism respecting the grading is not necessarily a morphism of Cox rings of type $\lambda$.

Example 2.14. For $X:=\mathbb{P}_{k}^{1}$, the Picard group $\operatorname{Pic}(X)$ is free of rank 1 , and $R \cong k\left[T_{0}, T_{1}\right]$ is a Cox ring of $X$ of type $\operatorname{id}_{\operatorname{Pic}(X)}$, where the $\operatorname{Pic}(X)$-grading is the usual $\mathbb{Z}$-grading by the total degree, identifying effective divisor classes with nonnegative integers. Mapping $T_{0}$ and $T_{1}$ to arbitrary linearly independent linear polynomials in $T_{0}, T_{1}$ defines an automorphism of $k\left[T_{0}, T_{1}\right]$ respecting the grading, but every Cox ring automorphism of $R$ in the sense of Definition 2.1. is multiplication by a scalar in $k^{\times}$.

We observe that every morphism $R \rightarrow R^{\prime}$ of Cox rings of type $\lambda$ in the sense of Definition 2.1 (cf. Remark 2.6) is an isomorphism, because it restricts to an isomorphism $R_{m} \rightarrow R_{m}^{\prime}$ for every $m \in M$, as it is compatible with the map div. 
Proposition 2.15. Every morphism of Cox sheaves of $X$ of type $\lambda$ is an isomorphism.

Proof. Let $\psi: \mathcal{R} \rightarrow \mathcal{R}^{\prime}$ be a morphism of Cox sheaves of $X$ of type $\lambda$. Let $\left\{\phi_{m, D}\right\}_{(m, D) \in M_{\lambda}}$ and $\left\{\phi_{m, D}^{\prime}\right\}_{(m, D) \in M_{\lambda}}$ be families of isomorphisms associated to $\mathcal{R}$ and $\mathcal{R}^{\prime}$, respectively. For every $m \in M$, fix a Cartier divisor $D_{m}$ such that $\left(m, D_{m}\right) \in M_{\lambda}$, and let $\alpha_{m} \in k[X]$ such that

$$
\left.\phi_{m, D_{m}}^{\prime} \circ \psi\right|_{\mathcal{R}_{m}} \circ \phi_{m, D_{m}}^{-1}=\alpha_{m} \operatorname{id}_{\mathcal{O}_{X}\left(D_{m}\right)}
$$

(cf. Lemma 2.4). Let $U$ be an open subset of $X$ that trivializes all elements in $\lambda(M)$. Fix $m \in M$. For every $s^{+} \in \mathcal{R}_{m}(U)$ and $s^{-} \in \mathcal{R}_{-m}(U)$,

$$
\begin{aligned}
& \beta^{-1} \alpha_{0} \phi_{m, D_{m}}\left(s^{+}\right) \phi_{-m, D_{-m}}\left(s^{-}\right)=\phi_{0, D_{0}}^{\prime}\left(\left.\psi\right|_{\mathcal{R}_{0}}\left(s^{+} s^{-}\right)\right) \\
& =\phi_{0, D_{0}}^{\prime}\left(\left.\left.\psi\right|_{\mathcal{R}_{m}}\left(s^{+}\right) \psi\right|_{\mathcal{R}_{-m}}\left(s^{-}\right)\right)=\left(\beta^{\prime}\right)^{-1} \alpha_{m} \alpha_{-m} \phi_{m, D_{m}}\left(s^{+}\right) \phi_{-m, D_{-m}}\left(s^{-}\right),
\end{aligned}
$$

where $\beta, \beta^{\prime} \in k^{\times}$are the unique constants such that

$$
\begin{aligned}
& \phi_{m, D_{m}}\left(s^{+}\right) \phi_{-m, D_{-m}}\left(s^{-}\right)=\beta \phi_{0, D_{0}}\left(s^{+} s^{-}\right), \\
& \phi_{m, D_{m}}^{\prime}\left(s^{+}\right) \phi_{-m, D_{-m}}^{\prime}\left(s^{-}\right)=\beta^{\prime} \phi_{0, D_{0}}^{\prime}\left(s^{+} s^{-}\right),
\end{aligned}
$$

respectively. Then $\alpha_{m} \alpha_{-m}=\beta^{\prime} \beta^{-1} \alpha_{0}$, as $\beta$ and $\beta^{\prime}$ do not depend on the choice of $s^{+}$and $s^{-}$. Since $\psi$ is a morphism of $\mathcal{O}_{X}$-algebras and both $\mathcal{R}_{0}$ and $\mathcal{R}_{0}^{\prime}$ are isomorphic to $\mathcal{O}_{X}$, we have $\psi(1)=1$, and $\left.\psi\right|_{\mathcal{R}_{0}}: \mathcal{R}_{0} \rightarrow \mathcal{R}_{0}^{\prime}$ is an isomorphism of $\mathcal{O}_{X}$-modules. Hence $\alpha_{0} \in k[X]^{\times}=k^{\times}$by Lemma 2.4 .

As a consequence of Proposition 2.15] and Lemma 2.4 every morphism of Cox sheaves of $X$ of type $\lambda$ induces a morphism of Cox rings of $X$ of type $\lambda$ between the rings of global sections in the sense of Definition 2.1. Hence, for every $\lambda$, there exists exactly one isomorphism class of Cox rings of $X$ of type $\lambda$ by Corollary 2.10 .

Proposition 2.16. Let $\mathcal{R}$ and $\mathcal{R}^{\prime}$ be two Cox sheaves of $X$ of type $\lambda$. If $M=M_{\mathrm{eff}}$, then every morphism $\mathcal{R}(X) \rightarrow \mathcal{R}^{\prime}(X)$ of Cox rings is induced by a unique morphism $\mathcal{R} \rightarrow \mathcal{R}^{\prime}$ of Cox sheaves.

Proof. Let $\left\{\phi_{m, D}\right\}_{(m, D) \in M_{\lambda}}$ and $\left\{\phi_{m, D}^{\prime}\right\}_{(m, D) \in M_{\lambda}}$ be families of isomorphisms associated with $\mathcal{R}$ and $\mathcal{R}^{\prime}$, respectively. For every $\left(m_{1}, D_{1}\right),\left(m_{2}, D_{2}\right) \in M_{\lambda}$, let $\beta_{m_{1}, m_{2} ; D_{1}, D_{2}}$ and $\beta_{m_{1}, m_{2} ; D_{1}, D_{2}}^{\prime}$ be the unique constants such that

$$
\begin{aligned}
& \phi_{m_{1}, D_{1}}\left(s_{1}\right) \phi_{m_{2}, D_{2}}\left(s_{2}\right)=\beta_{m_{1}, m_{2} ; D_{1}, D_{2}} \phi_{m_{1}+m_{2}, D_{1}+D_{2}}\left(s_{1} s_{2}\right), \\
& \phi_{m_{1}, D_{1}}^{\prime}\left(s_{1}^{\prime}\right) \phi_{m_{2}, D_{2}}^{\prime}\left(s_{2}^{\prime}\right)=\beta_{m_{1}, m_{2} ; D_{1}, D_{2}}^{\prime} \phi_{m_{1}+m_{2}, D_{1}+D_{2}}^{\prime}\left(s_{1}^{\prime} s_{2}^{\prime}\right)
\end{aligned}
$$

for all $s_{i} \in \mathcal{R}_{m_{i}}(U)$ and $s_{i}^{\prime} \in \mathcal{R}_{m_{i}}^{\prime}(U), i \in\{1,2\}$, and all open subsets $U$ of $X$.

Let $\psi: \mathcal{R}(X) \rightarrow \mathcal{R}^{\prime}(X)$ be a morphism of Cox rings of type $\lambda$. By definition of morphism of Cox rings of $X$ of type $\lambda$ (cf. Remark 2.6), for every $(m, D) \in M_{\lambda}$ such that $\lambda(m)$ is effective, we have $\left.\psi\right|_{\mathcal{R}(X)_{m}}=\alpha_{m, D}\left(\phi_{m, D}^{\prime}\right)^{-1} \circ \phi_{m, D}: \mathcal{R}(X)_{m} \rightarrow$ $\mathcal{R}^{\prime}(X)_{m}$ for a constant $\alpha_{m, D} \in k^{\times}$. Moreover,

$$
\alpha_{m_{1}+m_{2}, D_{1}+D_{2}}=\alpha_{m_{1}, D_{1}} \alpha_{m_{2}, D_{2}} \beta_{m_{1}, m_{2} ; D_{1}, D_{2}}\left(\beta_{m_{1}, m_{2} ; D_{1}, D_{2}}^{\prime}\right)^{-1}
$$

for all $\left(m_{1}, D_{1}\right),\left(m_{2}, D_{2}\right) \in M_{\lambda}$ as above, as $\psi$ is compatible with the multiplication in $\mathcal{R}(X)$ and in $\mathcal{R}^{\prime}(X)$.

For every $m \in M$, write $m=m_{1}-m_{2}$ with $m_{i} \in M$ such that $\lambda\left(m_{i}\right)$ is effective, choose Cartier divisors $D_{i}$ such that $\left(m_{i}, D_{i}\right) \in M_{\lambda}, i \in\{1,2\}$, and let $D:=D_{1}-D_{2}$. The isomorphisms

$$
\alpha_{m_{1}, D_{1}} \alpha_{m_{2}, D_{2}}^{-1} \beta_{m, m_{2} ; D, D_{2}}^{-1} \beta_{m, m_{2} ; D, D_{2}}^{\prime} \phi_{m, D}^{\prime}{ }^{-1} \circ \phi_{m, D}: \mathcal{R}_{m} \rightarrow \mathcal{R}_{m}^{\prime}
$$

define a morphism of Cox sheaves $\mathcal{R} \rightarrow \mathcal{R}^{\prime}$ that induces $\psi$ at the level of global sections. 
Proposition 2.17. Let $\mathcal{R}$ be a Cox sheaf of $X$ of type $\lambda$. For every $h \in \widehat{M}(k)=$ $\operatorname{Hom}\left(M, k^{\times}\right)$, let $\psi_{h}: \mathcal{R} \rightarrow \mathcal{R}$ be the map defined as scalar multiplication by $h(m)$ on $\mathcal{R}_{m}$ for all $m \in M$. Then $h \mapsto \psi_{h}$ defines an isomorphism between $\widehat{M}(k)$ and the group of Cox sheaf automorphisms of $\mathcal{R}$.

Proof. By Lemma 2.4, a Cox sheaf automorphism $\psi$ of $\mathcal{R}$ must be scalar multiplication by some $h_{m} \in k^{\times}$on each homogeneous part $\mathcal{R}_{m}$. Moreover, $h_{m} h_{m^{\prime}}=h_{m+m^{\prime}}$ for all $m, m^{\prime} \in M$ as $\psi$ is compatible with the multiplication in $\mathcal{R}$. So, $m \mapsto h_{m}$ defines a group homomorphism $M \rightarrow k^{\times}$, and hence an element $h \in \widehat{M}(k)$ such that $\psi=\psi_{h}$.

As a consequence of Propositions [2.15, 2.16 and 2.17, the group of Cox ring automorphisms of a Cox ring of $X$ of type $\lambda$ is isomorphic to $\widehat{M_{\mathrm{eff}}}(k)$.

Proposition 2.18. If $\pi: Y \rightarrow X$ is an $X$-torsor under $\widehat{M}$ of type $\lambda$, then $\pi_{*} \mathcal{O}_{Y}$ is a Cox sheaf of $X$ of type $\lambda$. Conversely, if $\mathcal{R}$ is a Cox sheaf of $X$ of type $\lambda$, then the relative spectrum $\operatorname{Spec}_{X} \mathcal{R} \rightarrow X$ is an $X$-torsor under $\widehat{M}$ of type $\lambda$.

Proof. Let $\pi: Y \rightarrow X$ be an $X$-torsor under $\widehat{M}$ of type $\lambda$. Let $\left\{U_{i}\right\}_{i}$ be an open covering of $X$ that trivializes $\pi$, and let $\left(\beta_{i, j}\right)_{i, j}$ with

$$
\beta_{i, j} \in \operatorname{Hom}\left(M, \mathcal{O}_{X}\left(U_{i} \cap U_{j}\right)^{\times}\right) \cong \widehat{M}\left(U_{i} \cap U_{j}\right)
$$

be a cocycle representing $[Y] \in H_{e ́ t}^{1}(X, \widehat{M})$. By definition of the type map (cf. Section [1.2), for every $m \in M$, there exists a Cartier divisor $D_{m}=\left\{\left(U_{i}, f_{i, m}\right)\right\}_{i}$ such that $\left[D_{m}\right]=\lambda(m)$ in $\operatorname{Pic}(X)$ and $\beta_{i, j}(m)=f_{i, m} f_{j, m}^{-1}$. The isomorphisms of $\mathcal{O}_{U_{i}}$-algebras

$$
\left.\pi_{*} \mathcal{O}_{Y}\right|_{U_{i}} \cong \mathcal{O}_{U_{i}}[M] \cong \bigoplus_{m \in M} f_{i, m}^{-1} \mathcal{O}_{U_{i}}
$$

glue over the cocycle $\left(\beta_{i, j}\right)_{i, j}$ to give an isomorphism of $\mathcal{O}_{X}$-modules

$$
\phi: \pi_{*} \mathcal{O}_{Y} \rightarrow \bigoplus_{m \in M} \mathcal{O}_{X}\left(D_{m}\right) .
$$

Hence $\pi_{*} \mathcal{O}_{Y}$ is an $M$-graded $\mathcal{O}_{X}$-algebra. For all $m \in M$, let $\phi_{m, D_{m}}$ be the isomorphism

$$
\left.\phi\right|_{\left(\pi_{*} \mathcal{O}_{Y}\right)_{m}}:\left(\pi_{*} \mathcal{O}_{Y}\right)_{m} \rightarrow \mathcal{O}_{X}\left(D_{m}\right)
$$

where $\left(\pi_{*} \mathcal{O}_{Y}\right)_{m}$ is the degree- $m$-part of $\pi_{*} \mathcal{O}_{Y}$. Let $U$ be an open subset of $X$, and $s_{1}, s_{2} \in \pi_{*} \mathcal{O}_{Y}(U)$ homogeneous of degree $m_{1}, m_{2}$, respectively. We observe that, for all $i$,

$$
\left.\phi\left(s_{1} s_{2}\right)\right|_{U \cap U_{i}}=\left.\left.f_{i, m_{1}+m_{2}}^{-1} f_{i, m_{1}} \phi\left(s_{1}\right)\right|_{U \cap U_{i}} f_{i, m_{2}} \phi\left(s_{2}\right)\right|_{U \cap U_{i}},
$$

where the product on the right is computed in $k(X)$. Since $f_{i, m_{1}+m_{2}}^{-1} f_{i, m_{1}} f_{i, m_{2}}$ belongs to $\mathcal{O}_{X}\left(U_{i}\right)^{\times}$for all $i$, and $f_{i, m_{1}+m_{2}}^{-1} f_{i, m_{1}} f_{i, m_{2}}=f_{j, m_{1}+m_{2}}^{-1} f_{j, m_{1}} f_{j, m_{2}}$ in $k(X)^{\times}$for all $i, j$ by restricting $\phi$ and $s_{1}, s_{2}$ to $U \cap U_{i} \cap U_{j}$, the element $\alpha:=$ $f_{i, m_{1}+m_{2}} f_{i, m_{1}}^{-1} f_{i, m_{2}}^{-1}$ belongs to $k[X]^{\times}=k^{\times}$, and

$$
\phi_{m_{1}, D_{m_{1}}}\left(s_{1}\right) \phi_{m_{2}, D_{m_{2}}}\left(s_{2}\right)=\alpha \phi_{m_{1}+m_{2}, D_{m_{1}+m_{2}}}\left(s_{1} s_{2}\right)
$$

for all $s_{1}, s_{2} \in \pi_{*} \mathcal{O}_{Y}(U)$ homogeneous of degree $m_{1}, m_{2}$, respectively.

Conversely, let $\mathcal{R}$ be a Cox sheaf of $X$ of type $\lambda$. The morphism $\pi: \operatorname{Spec}_{X} \mathcal{R} \rightarrow X$ induced by $\mathcal{O}_{X} \subseteq \mathcal{R}$ is surjective. For every open subset $U$ of $X$ that trivializes all elements of $\lambda(M)$, there are isomorphisms

$$
\left.\mathcal{R}\right|_{U} \cong \mathcal{O}_{U}[M] \cong \bigoplus_{m \in M} \mathcal{O}_{U}
$$

Therefore, $\mathcal{R}$ is locally free as an $\mathcal{O}_{X}$-module and locally finitely generated as an $\mathcal{O}_{X}$-algebra, and the morphism $\pi$ is flat and of finite type. Moreover, $\operatorname{Spec}_{X} \mathcal{R}$ is 
locally isomorphic to $\widehat{M}_{X} \cong \operatorname{Spec}_{X} \mathcal{O}_{X}[M]$ with the natural action of $\widehat{M}_{X}$ on itself. Hence $\pi$ is an $X$-torsor under $\widehat{M}$.

Let $\left\{U_{i}\right\}_{i}$ be an open covering that trivializes all elements of $\lambda(M)$. Then it trivializes $\pi$. Let $\left(\beta_{i, j}\right)_{i, j}$ with

$$
\beta_{i, j} \in \operatorname{Hom}\left(M, \mathcal{O}_{X}\left(U_{i} \cap U_{j}\right)^{\times}\right)
$$

be a cocycle representing the class of $\operatorname{Spec}_{X} \mathcal{R}$ in $H_{e t t}^{1}\left(X, \widehat{M}_{X}\right)$. By definition of the type map, the cocycle $\left(\beta_{i, j}(m)\right)_{i, j}$ represents the isomorphism class of $\mathcal{R}_{m}$ in $\operatorname{Pic}(X)$ for all $m \in M$. Since $\mathcal{R}$ is a Cox sheaf of type $\lambda$, for every $m \in M$, there is an isomorphism $\mathcal{R}_{m} \cong \mathcal{O}_{X}\left(D_{m}\right)$, where $D_{m}$ is a Cartier divisor such that $\left[D_{m}\right]=\lambda(m)$. Therefore, the cocycle $\left(\beta_{i, j}(m)\right)_{i, j}$ represents $\lambda(m)$ in $\operatorname{Pic}(X)$, and type $\left(\left[\operatorname{Spec}_{X} \mathcal{R}\right]\right)=\lambda$.

\section{OVER NONCLOSED FIELDS}

Let $k$ be a field. We fix a separable closure $\bar{k}$ of $k$, with Galois group $\mathfrak{g}:=$ $\operatorname{Gal}(\bar{k} / k)$, and every algebraic extension of $k$ mentioned later is contained in $\bar{k}$. In this section, $X$ always denotes a $k$-variety such that $X_{\bar{k}}:=X \times_{\operatorname{Spec} k} \operatorname{Spec} \bar{k}$ has only constant invertible regular functions (i.e., $\bar{k}\left[X_{\bar{k}}\right]^{\times}=\bar{k}^{\times}$, where $\bar{k}\left[X_{\bar{k}}\right]:=$ $\left.H^{0}\left(X_{\bar{k}}, \mathcal{O}_{X_{\bar{k}}}\right)\right)$.

The action of $\mathfrak{g}$ on $\bar{k}$ induces an action on $A \otimes_{k} \bar{k}$ (with $\mathrm{g} \in \mathfrak{g}$ acting via $\operatorname{id}_{A} \otimes \mathrm{g}$ ) for every $k$-algebra $A$, and similarly on $\mathcal{O}_{X_{\bar{k}}} \cong \mathcal{O}_{X} \otimes_{k} \bar{k}$. The action of an element $\mathrm{g} \in \mathfrak{g}$ on $X_{\bar{k}}$ is the one induced by the action of $\mathrm{g}^{-1}$ on $\mathcal{O}_{X_{\bar{k}}}$. For every $\mathrm{g} \in \mathfrak{g}$, we denote by $\mathrm{g} D$ the natural Galois action on a divisor $D \in \operatorname{CaDiv}\left(X_{\bar{k}}\right)$, by $\mathrm{g}(f)$ the natural Galois action on an element $f \in \bar{k}(X)$. All these actions are continuous (with respect to the Krull topology on $\mathfrak{g}$ and the discrete topology on the other objects). We will denote by $\mathrm{g} * s$ an action of $\mathrm{g} \in \mathfrak{g}$ on a section $s$ of a Cox sheaf (or an element $s$ of a Cox ring) of $X_{\bar{k}}$.

From here on, $M$ denotes a $\mathfrak{g}$-module that is finitely generated as an abelian group, and whose torsion is coprime to the characteristic of $k$. Let $\lambda: M \rightarrow \operatorname{Pic}\left(X_{\bar{k}}\right)$ be a homomorphism of $\mathfrak{g}$-modules. Let $\widehat{M}:=\operatorname{Spec} \bar{k}[M]^{\mathfrak{g}}$ be the quasitorus dual to $M$ under the antiequivalence of categories recalled in Section 1.2 Here, $\bar{k}[M]^{\mathfrak{g}}$ denotes the subring of $\mathfrak{g}$-invariant elements of $\bar{k}[M]$. As in Remark 2.13, let $M_{\text {eff }}$ be the subgroup of $M$ generated by the elements $m$ such that $\lambda(m)$ is effective in $\operatorname{Pic}\left(X_{\bar{k}}\right)$.

Definition 3.1. A continuous $\mathfrak{g}$-action on a Cox $\operatorname{ring} R$ of $X_{\bar{k}}$ of type $\lambda$ is called natural if $\operatorname{div}(\mathrm{g} * s)=\mathrm{g} \operatorname{div}(s)$ for all nonzero homogeneous $s \in R$ and all $\mathrm{g} \in \mathfrak{g}$. A $\mathfrak{g}$-equivariant Cox ring of $X_{\bar{k}}$ of type $\lambda$ is a Cox ring of $X_{\bar{k}}$ of type $\lambda$ with a natural $\mathfrak{g}$-action.

A continuous $\mathfrak{g}$-action on a Cox sheaf $\mathcal{R}$ of $X_{\bar{k}}$ of type $\lambda$ is called natural if, given an associated family of isomorphisms $\left\{\phi_{m, D}\right\}_{(m, D) \in M_{\lambda}}$, for every $\mathrm{g} \in \mathfrak{g}$ and $(m, D) \in M_{\lambda}$, the automorphism of $\mathcal{R}$ defined by the action of $\mathrm{g}$ restricts to an isomorphism $\mathcal{R}_{m} \rightarrow \mathcal{R}_{\mathrm{g} m}$ such that $\mathrm{g}^{-1} \circ \phi_{\mathrm{g} m, \mathrm{~g} D} \circ \mathrm{g} \circ \phi_{m, D}^{-1}$ is an automorphism of $\mathcal{O}_{X_{\bar{k}}}(D)$. A $\mathfrak{g}$-equivariant Cox sheaf of $X_{\bar{k}}$ of type $\lambda$ is a Cox sheaf of $X_{\bar{k}}$ of type $\lambda$ with a natural $\mathfrak{g}$-action.

A morphism of $\mathfrak{g}$-equivariant Cox sheaves (rings) of $X_{\bar{k}}$ of type $\lambda$ is a $\mathfrak{g}$-equivariant morphism of Cox sheaves (respectively, rings) of $X_{\bar{k}}$ of type $\lambda$.

Remark 3.2. Using the equivalent definition of Cox ring provided by Proposition 2.3. a continuous $\mathfrak{g}$-action on a Cox ring $R$ of $X_{\bar{k}}$ of type $\lambda$ is natural if and only if, given an associated family of isomorphisms $\left\{\phi_{m, D}\right\}_{(m, D) \in M_{\lambda}}$, for every $\mathrm{g} \in \mathfrak{g}$ and $(m, D) \in M_{\lambda}$, the automorphism of $R$ defined by the action of $\mathrm{g}$ restricts to 
an isomorphism $R_{m} \rightarrow R_{\mathrm{g} m}$ such that $\mathrm{g}^{-1} \circ \phi_{\mathrm{g} m, \mathrm{~g} D} \circ \mathrm{g} \circ \phi_{m, D}^{-1}=\alpha \operatorname{id}_{H^{0}\left(X_{\bar{k}}, \mathcal{O}_{X_{\bar{k}}}(D)\right)}$ with $\alpha \in \bar{k}^{\times}$. We observe that a natural $\mathfrak{g}$-action on a Cox sheaf $\mathcal{R}$ of $X_{\bar{k}}$ of type $\lambda$ induces a natural $\mathfrak{g}$-action on the Cox $\operatorname{ring} \mathcal{R}\left(X_{\bar{k}}\right)$ of $X_{\bar{k}}$ of type $\lambda$.

Definition 3.3. A Cox ring of $X$ of type $\lambda$ is a $k$-algebra $R$ together with a structure of Cox ring of $X_{\bar{k}}$ of type $\lambda$ on $R_{\bar{k}}:=R \otimes_{k} \bar{k}$ such that $R_{\bar{k}}$ with the induced action of $\mathfrak{g}$ is a $\mathfrak{g}$-equivariant Cox ring of $X_{\bar{k}}$. A Cox sheaf of $X$ of type $\lambda$ is a sheaf $\mathcal{R}$ of $\mathcal{O}_{X}$-algebras together with a structure of Cox sheaf of $X_{\bar{k}}$ of type $\lambda$ on $\mathcal{R}_{\bar{k}}:=\mathcal{R} \otimes_{k} \bar{k}$ such that $\mathcal{R}_{\bar{k}}$ with the induced action of $\mathfrak{g}$ is a $\mathfrak{g}$-equivariant Cox sheaf of $X_{\bar{k}}$.

A morphism of Cox rings of $X$ of type $\lambda$ is a morphism of $k$-algebras $\psi: R \rightarrow R^{\prime}$ such that $\psi \otimes \operatorname{id}_{\bar{k}}: R_{\bar{k}} \rightarrow R_{\bar{k}}^{\prime}$ is a morphism of Cox rings of $X_{\bar{k}}$ of type $\lambda$. A morphism of Cox sheaves of $X$ of type $\lambda$ is a morphism of $\mathcal{O}_{X}$-algebras $\psi: \mathcal{R} \rightarrow \mathcal{R}^{\prime}$ such that $\psi \otimes \operatorname{id}_{\bar{k}}: \mathcal{R}_{\bar{k}} \rightarrow \mathcal{R}_{\bar{k}}^{\prime}$ is a morphism of Cox sheaves of $X_{\bar{k}}$ of type $\lambda$.

Proposition 3.4. The covariant functors

$$
\begin{aligned}
\{\text { Cox rings of } X \text { of type } \lambda\} & \longrightarrow\left\{\begin{array}{c}
\mathfrak{g} \text {-equivariant Cox rings } \\
\text { of } X_{\bar{k}} \text { of type } \lambda
\end{array}\right\} \\
R & \longmapsto R_{\bar{k}}
\end{aligned}
$$

and

$$
\begin{aligned}
\{\text { Cox sheaves of } X \text { of type } \lambda\} & \longrightarrow\left\{\begin{array}{c}
\mathfrak{g} \text {-equivariant Cox sheaves } \\
\text { of } X_{\bar{k}} \text { of type } \lambda
\end{array}\right\} \\
\mathcal{R} & \longmapsto \mathcal{R}_{\bar{k}}
\end{aligned}
$$

are equivalences of categories, with inverse functor $H^{0}(\mathfrak{g}, \cdot)$.

Proof. Let $R$ be a $\mathfrak{g}$-equivariant Cox ring of $X_{\bar{k}}$ of type $\lambda$, and $R^{\mathfrak{g}}:=H^{0}(\mathfrak{g}, R)$ its subring of $\mathfrak{g}$-invariant elements. Since the action of $\mathfrak{g}$ on $R$ is continuous, there is an isomorphism $R^{\mathfrak{g}} \otimes_{k} \bar{k} \cong R$ by Mil12, Proposition 16.15]. Similarly, if $\mathcal{R}$ is a $\mathfrak{g}$-equivariant Cox sheaf of $X_{\bar{k}}$ of type $\lambda$, then the sheaf $\mathcal{R}^{\mathfrak{g}}$ defined by $\mathcal{R}^{\mathfrak{g}}(U):=$ $H^{0}\left(\mathfrak{g}, \mathcal{R}\left(U_{\bar{k}}\right)\right)$ for all open subsets $U$ of $X$ is a Cox sheaf of $X$ of type $\lambda$. Moreover, if $\psi: \mathcal{R} \rightarrow \mathcal{R}^{\prime}$ is a morphism of $\mathfrak{g}$-equivariant Cox sheaves of $X_{\bar{k}}$ of type $\lambda$, then $\psi\left(\mathcal{R}\left(U_{\bar{k}}\right)^{\mathfrak{g}}\right) \subseteq \mathcal{R}^{\prime}\left(U_{\bar{k}}\right)^{\mathfrak{g}}$ for every open subset $U$ of $X$. Hence $\psi$ restricts to a unique morphism $\psi^{\mathfrak{g}}: \mathcal{R}^{\mathfrak{g}} \rightarrow \mathcal{R}^{\prime \mathfrak{g}}$ of Cox sheaves of $X$ of type $\lambda$ such that $\psi=\psi^{\mathfrak{g}} \otimes \mathrm{id}_{\bar{k}}$ under the identifications $\mathcal{R} \cong\left(\mathcal{R}^{\mathfrak{g}}\right)_{\bar{k}}$ and $\mathcal{R}^{\prime} \cong\left(\mathcal{R}^{\prime \mathfrak{g}}\right)_{\bar{k}}$.

Proposition 3.5. Let $\mathcal{R}$ be a Cox sheaf of $X_{\bar{k}}$ of type $\lambda$. If $M=M_{\mathrm{eff}}$, then every natural $\mathfrak{g}$-action on $\mathcal{R}\left(X_{\bar{k}}\right)$ is induced by a natural $\mathfrak{g}$-action on $\mathcal{R}$.

Proof. Assume that $\mathcal{R}\left(X_{\bar{k}}\right)$ is endowed with a natural $\mathfrak{g}$-action. Let $\phi_{m, D}: \mathcal{R}_{m} \rightarrow$ $\mathcal{O}_{X_{\bar{k}}}(D)$, for $(m, D) \in M_{\lambda}$, be a family of isomorphisms associated with $\mathcal{R}$. For every $\mathrm{g} \in \mathfrak{g}$ and $(m, D) \in M_{\lambda}$ such that $\lambda(m)$ is effective in $\operatorname{Pic}\left(X_{\bar{k}}\right)$, let $\alpha_{\mathrm{g}, m, D} \in \bar{k}^{\times}$ be the unique constant such that

$$
\phi_{\mathrm{g} m, \mathrm{~g} D} \circ \mathrm{g}=\alpha_{\mathrm{g}, m, D} \mathrm{~g} \circ \phi_{m, D} .
$$

For an arbitrary $(m, D) \in M_{\lambda}$, let $\left(m^{+}, D^{+}\right),\left(m^{-}, D^{-}\right) \in M_{\lambda}$ such that $m=$ $m^{+}-m^{-}, D=D^{+}-D^{-}$, and $\lambda\left(m^{+}\right)$and $\lambda\left(m^{-}\right)$are both effective in $\operatorname{Pic}\left(X_{\bar{k}}\right)$. We define

$$
\alpha_{\mathrm{g}, m, D}:=\alpha_{\mathrm{g}, m^{+}, D^{+}} \alpha_{\mathrm{g}, m^{-}, D^{-}}^{-1} \beta \mathrm{g}\left(\gamma^{-1}\right),
$$

where $\beta, \gamma \in \bar{k}^{\times}$are the unique constants such that

$$
\begin{gathered}
\phi_{m, D}(s) \phi_{m^{-}, D^{-}}\left(s^{\prime}\right)=\gamma \phi_{m^{+}, D^{+}}\left(s s^{\prime}\right) \\
\phi_{\mathrm{g} m, \mathrm{~g} D}(\mathrm{~g} * s) \phi_{\mathrm{g} m^{-}, \mathrm{g} D^{-}}\left(\mathrm{g} * s^{\prime}\right)=\beta \phi_{\mathrm{g} m^{+}, \mathrm{g} D^{+}}\left(\mathrm{g} *\left(s s^{\prime}\right)\right)
\end{gathered}
$$


for all $s \in \mathcal{R}\left(X_{\bar{k}}\right)_{m}$ and $s^{\prime} \in \mathcal{R}\left(X_{\bar{k}}\right)_{m^{-}}$. The constant $\alpha_{\mathrm{g}, m, D}$ does not depend on the choice of $\left(m^{+}, D^{+}\right)$and $\left(m^{-}, D^{-}\right)$.

For every homogeneous section $s$ of $\mathcal{R}$ and $\mathrm{g} \in \mathfrak{g}$, let $\mathrm{g} * s:=\alpha_{\mathrm{g}, m, D}\left(\phi_{\mathrm{g} m, \mathrm{~g} D}^{-1} \circ\right.$ $\left.\mathrm{g} \circ \phi_{m, D}\right)(s)$ for all $(m, D) \in M_{\lambda}$ such that $s$ has degree $m$. This definition does not depend on the choice of the representative $D$ for $\lambda(m)$ and induces a natural action of $\mathfrak{g}$ on $\mathcal{R}$ in the sense of Definition 3.1.

Corollary 3.6. If $\mathcal{R}$ is a Cox sheaf of $X$ of type $\lambda$, then $\mathcal{R}(X)$ is a Cox ring of $X$ of type $\lambda$. Moreover, if $M=M_{\mathrm{eff}}$, the covariant functor

$$
\begin{aligned}
\{\text { Cox sheaves of } X \text { of type } \lambda\} & \longrightarrow\{\text { Cox rings of } X \text { of type } \lambda\} \\
\mathcal{R} & \longmapsto \mathcal{R}(X)
\end{aligned}
$$

is an equivalence of categories.

Proof. Let $\mathcal{R}$ be a Cox sheaf of $X$ of type $\lambda$. By Definition 3.3 , $\mathcal{R}_{\bar{k}}$ is a $\mathfrak{g}$-equivariant Cox sheaf of $X_{\bar{k}}$. Hence $\mathcal{R}_{\bar{k}}\left(X_{\bar{k}}\right)$ is a Cox ring of $X_{\bar{k}}$ of type $\lambda$, and the induced $\mathfrak{g}$-action turns it into a $\mathfrak{g}$-equivariant Cox ring of type $\lambda$. Then the subring of $\mathfrak{g}$ invariant elements $\mathcal{R}(X)=\mathcal{R}_{\bar{k}}\left(X_{\bar{k}}\right)^{\mathfrak{g}}$ is a Cox ring of $X$ of type $\lambda$ by Proposition 3.4

Assume now that $M=M_{\text {eff }}$. The functor is essentially surjective by Propositions 2.11 3.5 and 3.4. We prove that it is fully faithful. Let $\mathcal{R}$ and $\mathcal{R}^{\prime}$ be Cox sheaves of $X$ of type $\lambda$. A morphism $\psi: \mathcal{R}(X) \rightarrow \mathcal{R}^{\prime}(X)$ of Cox rings of $X$ of type $\lambda$ induces a morphism of $\mathfrak{g}$-equivariant Cox rings $\mathcal{R}(X)_{\bar{k}} \rightarrow \mathcal{R}^{\prime}(X)_{\bar{k}}$ that extends to a unique isomorphism of Cox sheaves $\bar{\psi}: \mathcal{R}_{\bar{k}} \rightarrow \mathcal{R}_{\bar{k}}^{\prime}$ by Propositions 2.16 and 2.15. Since $\mathrm{g}^{-1} \circ \bar{\psi}^{-1} \circ \mathrm{g} \circ \bar{\psi}$ is an automorphism on $\mathcal{R}_{\bar{k}}$ that induces the identity on $\mathcal{R}_{\bar{k}}\left(X_{\bar{k}}\right)$ for every $\mathrm{g} \in \mathfrak{g}$, by the uniqueness statement of Proposition $2.16, \mathrm{~g}^{-1} \circ \bar{\psi}^{-1} \circ \mathrm{g} \circ \bar{\psi}$ is the identity as well, which means that $\bar{\psi}$ is $\mathfrak{g}$-equivariant, and hence restricts to an isomorphism $\mathcal{R} \cong \mathcal{R}^{\prime}$ inducing $\psi$.

We apply descent theory (see [Ser02, §III.1] or [Mil12, §16]) to classify Cox rings and Cox sheaves of $X$ up to isomorphism. Recall that by Corollary 2.10, every two Cox sheaves of $X$ of type $\lambda$ become isomorphic after the field extension $k \subseteq \bar{k}$. Moreover, if $\psi$ is an isomorphism of Cox sheaves of type $\lambda$ between two $\mathfrak{g}$-equivariant Cox sheaves of $X_{\bar{k}}$ of type $\lambda$, then ${ }^{\mathrm{g}} \psi:=\mathrm{g} \circ \psi \circ \mathrm{g}^{-1}$ is another isomorphism for all $\mathrm{g} \in \mathfrak{g}$, and sending $\mathrm{g}$ to the automorphism $\psi^{-1} \circ{ }^{\mathrm{g}} \psi$ defines a map $\mathfrak{g} \rightarrow \widehat{M}(\bar{k})$ by Proposition 2.17, which turns out to be a 1-cocycle.

Proposition 3.7. Assume that $X$ has a Cox sheaf $\mathcal{R}$ of type $\lambda$. Sending a Cox sheaf $\mathcal{R}^{\prime}$ of $X$ of type $\lambda$ with a Cox sheaf isomorphism $\psi: \mathcal{R}_{\bar{k}} \rightarrow \mathcal{R}_{\bar{k}}^{\prime}$ to the class of the cocycle

$$
\mathfrak{g} \rightarrow \operatorname{Aut}\left(\mathcal{R}_{\bar{k}}\right) \cong \widehat{M}(\bar{k}), \quad \mathrm{g} \mapsto \psi^{-1} \circ{ }^{\mathrm{g}} \psi
$$

defines an bijective map from the set of isomorphism classes of Cox sheaves of $X$ of type $\lambda$ to $H_{e ́ t}^{1}(k, \widehat{M})$.

Proof. The class of the cocycle $\mathrm{g} \mapsto \psi^{-1} \circ{ }^{\mathrm{g}} \psi$ does not depend on the choice of $\psi$. Moreover, if $\mathcal{R}^{\prime}, \mathcal{R}^{\prime \prime}$ are two Cox sheaves of $X$ of type $\lambda$, and $\psi^{\prime}: \mathcal{R}_{\bar{k}} \rightarrow \mathcal{R}_{\bar{k}}^{\prime}$, $\psi^{\prime \prime}: \mathcal{R}_{\bar{k}} \rightarrow \mathcal{R}_{\bar{k}}^{\prime \prime}$ are two Cox sheaf isomorphisms, the associated cocycles have the same class in $H_{e ́ t}^{1}(k, \widehat{M})$ if and only if there is an isomorphism of Cox sheaves $\mathcal{R}^{\prime} \rightarrow \mathcal{R}^{\prime \prime}$. Hence the map in the statement is injective.

For surjectivity, note that a cocycle $\sigma: \mathfrak{g} \rightarrow \widehat{M}(\bar{k})$ defines a twisted action

$$
\mathfrak{g} \times \mathcal{R}_{\bar{k}} \rightarrow \mathcal{R}_{\bar{k}}, \quad(\mathrm{~g}, s) \mapsto \sigma_{\mathrm{g}}(\mathrm{g}(s)):=\left(\psi_{\sigma_{\mathrm{g}}} \circ\left(\mathrm{id}_{\mathcal{R}} \otimes \mathrm{g}\right)\right)(s),
$$


where $\psi_{\sigma_{\mathrm{g}}}$ is the automorphism defined in Proposition 2.17. Since

$$
H^{1}(\mathfrak{g}, \widehat{M}(\bar{k}))=\underset{k^{\prime} / k}{\lim _{\longrightarrow}} H^{1}\left(\operatorname{Gal}\left(k^{\prime} / k\right), \widehat{M}\left(k^{\prime}\right)\right),
$$

where the direct limit is taken over the finite Galois extensions $k^{\prime}$ of $k$ inside $\bar{k}$, the twisted action defined by $\sigma$ is continuous, and hence it is a natural $\mathfrak{g}$-action on $\mathcal{R}_{\bar{k}}$ according to Definition 3.1. Let $\mathcal{R}^{\sigma}$ be the sheaf of invariants of this action. By Proposition 3.4, $\mathcal{R}^{\sigma}$ is a Cox sheaf of $X$ of type $\lambda$ such that $\mathcal{R}_{\bar{k}} \cong \mathcal{R}^{\sigma} \otimes_{k} \bar{k}$. The cocycle associated with this isomorphism is $\left(\mathrm{id}_{\mathcal{R}_{\bar{k}}} \circ \psi_{\sigma_{\mathrm{g}}} \circ \mathrm{g} \circ \mathrm{id}_{\mathcal{R}_{\bar{k}}} \circ \mathrm{g}^{-1}\right)_{\mathrm{g} \in \mathfrak{g}}=\sigma$.

As a consequence of Corollary 3.6 and Proposition 3.7, the map in Proposition 3.7 defines a bijection between the set of isomorphism classes of Cox rings of $X$ of type $\lambda$ and $H_{e ́ t}^{1}\left(k, \widehat{M_{\text {eff }}}\right)$.

The inverse map to the bijection in Proposition 3.7 is obtained by twisting a Cox sheaf of $X$ of type $\lambda$ by cocycles. Therefore, we introduce the notion of twisted Cox sheaf.

Definition 3.8. For every Cox sheaf $\mathcal{R}$ of $X$ of type $\lambda$ and every cocycle $\sigma: \mathfrak{g} \rightarrow$ $\widehat{M}(\bar{k})$, we denote by $\mathcal{R}^{\sigma}$ the twisted Cox sheaf constructed in the proof of Proposition 3.7.

Proposition 3.9. If $\mathcal{R}$ is a Cox sheaf of $X$ of type $\lambda$, then $\operatorname{Spec}_{X} \mathcal{R}$ is an $X$-torsor of type $\lambda$. Moreover, for each cocycle $\sigma: \mathfrak{g} \rightarrow \widehat{M}(\bar{k})$, the $X$-torsor $\operatorname{Spec}_{X} \mathcal{R}^{\sigma}$ has class $\left[\operatorname{Spec}_{X} \mathcal{R}\right]-[\sigma]$ in $H_{e ́ t}^{1}\left(X, \widehat{M}_{X}\right)$.

Proof. Let $\mathcal{R}$ be a Cox sheaf of $X$ of type $\lambda$. Since $\mathcal{R}_{\bar{k}}$ is a $\mathfrak{g}$-equivariant Cox sheaf of $X_{\bar{k}}$, the action of Spec $\bar{k}[M]$ on $\operatorname{Spec}_{X_{\bar{k}}} \mathcal{R}_{\bar{k}}$ induced by the $M$-grading on $\mathcal{R}_{\bar{k}}$ descends to an action of $\widehat{M}=\operatorname{Spec} \bar{k}[M]^{\mathfrak{g}}$ on $\operatorname{Spec}_{X} \mathcal{R}$, and the canonical morphism $\operatorname{Spec}_{X} \mathcal{R} \rightarrow X$ is an $X$-torsor of type $\lambda$ by Proposition 2.18 and fpqc-descent.

Let $\sigma: \mathfrak{g} \rightarrow \widehat{M}(\bar{k})$ be a cocycle. By Proposition 3.7, the twisted Cox sheaf $\mathcal{R}^{\sigma}$ corresponds to the $\mathfrak{g}$-equivariant Cox sheaf $\mathcal{R}_{\bar{k}}$ of $X_{\bar{k}}$ with the twisted action of $\mathfrak{g}$ given by $(\mathrm{g}, s) \mapsto \sigma_{\mathrm{g}}(\mathrm{g}(s))$ under the bijection of Proposition 3.4. Thus, $\mathrm{Spec}_{X} \mathcal{R}^{\sigma}$ is obtained by Galois descent from $\operatorname{Spec}_{X_{\bar{k}}} \mathcal{R}_{\bar{k}}$ with the twisted $\mathfrak{g}$-action $(\mathrm{g}, x) \mapsto$ $\sigma_{\mathrm{g}}^{-1}(\mathrm{~g}(x))$. Therefore, $\left[\operatorname{Spec}_{X} \mathcal{R}^{\sigma}\right]=\left[\operatorname{Spec}_{X} \mathcal{R}\right]-[\sigma]$ in $H_{e ́ t}^{1}\left(X, \widehat{M}_{X}\right)$; see [Sko01, Example 2, p. 21].

Proposition 3.10. If $\pi: Y \rightarrow X$ is an $X$-torsor of type $\lambda$, then $\pi_{*} \mathcal{O}_{Y}$ is a Cox sheaf of $X$ of type $\lambda, \mathcal{O}_{Y}(Y)$ is a Cox ring of $X$ of type $\lambda$, and $\operatorname{Spec}_{X} \pi_{*} \mathcal{O}_{Y} \cong Y$.

Proof. Let $\pi: Y \rightarrow X$ be an $X$-torsor of type $\lambda$. Let $\bar{\pi}: Y_{\bar{k}} \rightarrow X_{\bar{k}}$ be the induced $X_{\bar{k}}$-torsor of type $\lambda$. Then $\bar{\pi}_{*} \mathcal{O}_{Y_{\bar{k}}}$ is a Cox sheaf of $X_{\bar{k}}$ of type $\lambda$ by Proposition 2.18. The induced $\mathfrak{g}$-action on $\bar{\pi}_{*} \mathcal{O}_{Y_{\bar{k}}}$ is a natural $\mathfrak{g}$-action in the sense of Definition 3.1. as it is continuous and compatible with the $\mathfrak{g}$-action on $M$. Hence $\pi_{*} \mathcal{O}_{Y}$ is a Cox sheaf of $X$ of type $\lambda$ according to Definition 3.3. and $\mathcal{O}_{Y}(Y)=\left(\pi_{*} \mathcal{O}_{Y}\right)(X)$ is a Cox ring of $X$ of type $\lambda$ by Corollary 3.6. Moreover, $\pi$ is affine by [MFK94, Proposition 0.7]. Hence $\operatorname{Spec}_{X} \pi_{*} \mathcal{O}_{Y} \cong Y$.

Corollary 3.11. The contravariant functor

$$
\begin{aligned}
\{\text { Cox sheaves of } X \text { of type } \lambda\} & \longrightarrow\{X \text {-torsors of type } \lambda\} \\
\mathcal{R} & \longmapsto \operatorname{Spec}_{X} \mathcal{R}
\end{aligned}
$$

is an anti-equivalence of categories, with inverse functor

$$
\begin{aligned}
\{X \text {-torsors of type } \lambda\} & \longrightarrow\{\text { Cox sheaves of } X \text { of type } \lambda\} \\
\pi: Y \rightarrow X & \longmapsto \pi_{*} \mathcal{O}_{Y} .
\end{aligned}
$$


Proof. By Propositions 3.9 and 3.10, both functors are well-defined and essentially surjective. Moreover, the functor $\mathrm{Spec}_{X}$ is fully faithful.

Proof of Theorem 1.1. Combine Corollary 3.6 with Corollary 3.11.

Now we discuss the functorial properties of Cox rings and Cox sheaves of varieties over an arbitrary field. We refer to [CTS87, §1.5] and [BH03, §6] for the analogous properties of torsors under quasitori and of Cox rings of identity type, respectively.

By CTS87, Proposition 1.5.2], the exact sequence (1.1), which classifies $X$ torsors under a quasitorus $G$, is functorial in $X$ and $G$, covariant in $G$ and contravariant in $X$. Therefore, if $M$ denotes the $\mathfrak{g}$-module dual to $G$ (i.e., the group of characters of $G_{\bar{k}}$ ), the exact sequence (1.1) is functorial in $X$ and $M$, contravariant with respect to both.

We consider first the functoriality with respect to morphisms of quasitori $G \rightarrow$ $G^{\prime}$, or equivalently, with respect to the induced morphisms of dual $\mathfrak{g}$-modules $\varphi: M^{\prime} \rightarrow M$.

If $\mathcal{R}$ is a Cox sheaf of $X$ of type $\lambda: M \rightarrow \operatorname{Pic}\left(X_{\bar{k}}\right)$, and $\left\{\phi_{m, D}\right\}_{(m, D) \in M_{\lambda}}$ is a family of isomorphisms associated with $\mathcal{R}_{\bar{k}}$, then the family of isomorphisms $\left\{\phi_{\varphi\left(m^{\prime}\right), D}\right\}_{\left(m^{\prime}, D\right) \in M_{\lambda \circ \varphi}^{\prime}}$ defines on $\bigoplus_{m^{\prime} \in M^{\prime}}\left(\mathcal{R}_{\bar{k}}\right)_{\varphi\left(m^{\prime}\right)}$ a structure of Cox sheaf of type $\lambda \circ \varphi$. The latter inherits from $\mathcal{R}_{\bar{k}}$ an action of $\mathfrak{g}$ that turns it into a $\mathfrak{g}$ equivariant Cox sheaf.

Definition 3.12. We define the pullback of $\mathcal{R}$ under $\varphi$ to be

$$
\varphi^{*} \mathcal{R}:=\left(\bigoplus_{m^{\prime} \in M^{\prime}}\left(\mathcal{R}_{\bar{k}}\right)_{\varphi\left(m^{\prime}\right)}\right)^{\mathfrak{g}} .
$$

The morphism $\varphi^{*} \mathcal{R}_{\bar{k}} \rightarrow \mathcal{R}_{\bar{k}}$ that restricts to the identity $\left(\varphi^{*} \mathcal{R}_{\bar{k}}\right)_{m^{\prime}}=\left(\mathcal{R}_{\bar{k}}\right)_{\varphi\left(m^{\prime}\right)}$ for all $m^{\prime} \in M^{\prime}$ is $\mathfrak{g}$-equivariant and descends to a morphism of $\mathcal{O}_{X}$-algebras

$$
\varphi^{*} \mathcal{R} \rightarrow \mathcal{R}
$$

Moreover, each morphism $\psi: \mathcal{R} \rightarrow \mathcal{R}^{\prime}$ of Cox sheaves of $X$ of type $\lambda$ pulls back under $\varphi$ to a morphism $\varphi^{*}(\psi): \varphi^{*} \mathcal{R} \rightarrow \varphi^{*} \mathcal{R}^{\prime}$ of Cox sheaves of $X$ of type $\lambda \circ \varphi$ such that the following diagram is commutative:

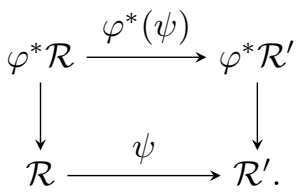

We show that the pullback of Cox sheaves under $\varphi$ corresponds to the pushforward of torsors defined in [Sko01, Example 3, p. 21] under the dual morphism of quasitori $\widehat{\varphi}: G \rightarrow G^{\prime}$.

Proposition 3.13. Let $\varphi: M^{\prime} \rightarrow M$ be a morphism of finitely generated $\mathfrak{g}$-modules whose torsion is coprime to the characteristic of $k$. Let $\mathcal{R}$ be a Cox sheaf of type $\lambda: M \rightarrow \operatorname{Pic}\left(X_{\bar{k}}\right)$. Then $\widehat{\varphi}_{*} \operatorname{Spec}_{X} \mathcal{R} \cong \operatorname{Spec}_{X} \varphi^{*} \mathcal{R}$.

Proof. Let $\left\{U_{i}\right\}_{i}$ be an affine étale covering of $X$ that trivializes the torsor $\operatorname{Spec}_{X} \mathcal{R}$. Let $U_{i, j}:=U_{i} \times_{X} U_{j}$, and let

$$
\beta_{i, j} \in \operatorname{Hom}_{\mathfrak{g}}\left(M, \mathcal{O}_{U_{i, j, \bar{k}}}\left(U_{i, j, \bar{k}}\right)^{\times}\right) \cong \widehat{M}\left(U_{i, j}\right)
$$

be a cocycle representing $\left[\operatorname{Spec}_{X} \mathcal{R}\right] \in H_{e ́ t}^{1}\left(X, \widehat{M}_{X}\right)$. Then the class $\widehat{\varphi}_{*}\left[\left(\beta_{i, j}\right)_{i, j}\right]$ of $\widehat{\varphi}_{*} \operatorname{Spec}_{X} \mathcal{R}$ in $H_{e t}^{1}\left(X, \widehat{M}_{X}^{\prime}\right)$ is represented by the cocycle $\left(\beta_{i, j} \circ \varphi\right)_{i, j}$. 
By definition, the torsor $\operatorname{Spec}_{X} \mathcal{R}$ is obtained by gluing

$$
\left\{\left(\operatorname{Spec}_{X} \mathcal{R}\right) \times_{X} U_{i} \cong \operatorname{Spec}_{U_{i}} \mathcal{O}_{U_{i, \bar{k}}}[M]^{\mathfrak{g}}\right\}_{i}
$$

over the isomorphisms

$$
\mathcal{O}_{U_{j, i, \bar{k}}}[M] \rightarrow \mathcal{O}_{U_{i, j, \bar{k}}}[M], \quad 1 \cdot m \mapsto \beta_{i, j}(m) \cdot m, \quad \forall m \in M .
$$

By definition of pullback, $\operatorname{Spec}_{X} \varphi^{*} \mathcal{R}$ is obtained by gluing

$$
\left\{\left(\operatorname{Spec}_{X} \varphi^{*} \mathcal{R}\right) \times_{X} U_{i} \cong \operatorname{Spec}_{U_{i}} \mathcal{O}_{U_{i, \bar{k}}}\left[M^{\prime}\right]^{\mathfrak{g}}\right\}_{i}
$$

over the isomorphisms

$$
\mathcal{O}_{U_{j, i, \bar{k}}}\left[M^{\prime}\right] \rightarrow \mathcal{O}_{U_{i, j, \bar{k}}}\left[M^{\prime}\right], \quad 1 \cdot m^{\prime} \mapsto \beta_{i, j}\left(\varphi\left(m^{\prime}\right)\right) \cdot m^{\prime}, \quad \forall m^{\prime} \in M^{\prime} .
$$

Hence the isomorphism class of $\operatorname{Spec}_{X} \varphi^{*} \mathcal{R}$ in $H_{e ́ t}^{1}\left(X, \widehat{M}^{\prime}{ }_{X}\right)$ is represented by the cocycle $\left(\beta_{i, j} \circ \varphi\right)_{i, j}$.

Now we consider the functoriality with respect to morphisms of $k$-varieties. Let $p: X^{\prime} \rightarrow X$ be a morphism of $k$-varieties and $\mathcal{R}$ a Cox sheaf of $X$ of type $\lambda: M \rightarrow$ $\operatorname{Pic}\left(X_{\bar{k}}\right)$. We denote by $p^{*}: \operatorname{Pic}\left(X_{\bar{k}}\right) \rightarrow \operatorname{Pic}\left(X_{\bar{k}}^{\prime}\right)$ the pullback of divisor classes under $p$. We recall that the property of being a torsor over $X$ under $G$ is stable under base extension. Therefore, $\operatorname{Spec}_{X^{\prime}} p^{*} \mathcal{R} \cong X^{\prime} \times_{X} \operatorname{Spec}_{X} \mathcal{R}$ is an $X^{\prime}$-torsor under $\widehat{M}$.

Proposition 3.14. Let $p: X^{\prime} \rightarrow X$ be a morphism of $k$-varieties, $p^{*}: \operatorname{Pic}\left(X_{\bar{k}}\right) \rightarrow$ $\operatorname{Pic}\left(X_{\bar{k}}^{\prime}\right)$ the induced pullback of divisor classes, and $\mathcal{R}$ a Cox sheaf of $X$ of type $\lambda: M \rightarrow \operatorname{Pic}\left(X_{\bar{k}}\right)$. Then $p^{*} \mathcal{R}$ is a Cox sheaf of $X^{\prime}$ of type $p^{*} \circ \lambda: M \rightarrow \operatorname{Pic}\left(X_{\bar{k}}^{\prime}\right)$.

Proof. If $\left\{\phi_{m, D}\right\}_{(m, D) \in M_{\lambda}}$ is a family of isomorphisms associated with the Cox sheaf $\mathcal{R}$, then the family of isomorphisms

$$
p^{*}\left(\phi_{m, D}\right): p^{*} \mathcal{R}_{m} \rightarrow p^{*} \mathcal{O}_{X^{\prime}}(D),
$$

for $(m, D) \in M_{\lambda}$, defines a structure of Cox sheaf of $X^{\prime}$ of type $p^{*} \circ \lambda$ on $p^{*} \mathcal{R}$, as $p^{*}$ commutes with direct limits.

Corollary 3.15. Let $p: X^{\prime} \rightarrow X$ be a morphism of $k$-varieties, and $\varphi: M^{\prime} \rightarrow M a$ morphism of finitely generated $\mathfrak{g}$-modules with torsion coprime to the characteristic of $k$.

(1) If $\mathcal{R}$ is a Cox sheaf of $X$ of type $\lambda: M \rightarrow \operatorname{Pic}\left(X_{\bar{k}}\right)$, then $p^{*} \varphi^{*} \mathcal{R}$ is a Cox sheaf of $X^{\prime}$ of type

$$
p^{*} \circ \lambda \circ \varphi: M^{\prime} \rightarrow \operatorname{Pic}\left(X_{k}^{\prime}\right) .
$$

(2) If $\psi: \mathcal{R} \rightarrow \mathcal{R}^{\prime}$ is a morphism of Cox sheaves of $X$ of type $\lambda$, then

$$
p^{*}\left(\varphi^{*}(\psi)\right): p^{*} \varphi^{*} \mathcal{R} \rightarrow p^{*} \varphi^{*} \mathcal{R}^{\prime}
$$

is a morphism of Cox sheaves of $X^{\prime}$ of type $p^{*} \circ \lambda \circ \varphi$.

(3) The double pullback $p^{*} \circ \varphi^{*}$ is a covariant functor from the category of Cox sheaves of $X$ of type $\lambda$ to the category of Cox sheaves of $X^{\prime}$ of type $p^{*} \circ \lambda \circ \varphi$.

(4) There is an isomorphism of functors between $p^{*} \circ \varphi^{*}$ and $\varphi^{*} \circ p^{*}$.

Proof. Parts (1) and (2) are a consequence of Proposition 3.14 and the discussion above.

For (3), we observe that $\varphi^{*}$ and $p^{*}$ are both covariant functors. For (4), we observe that $p^{*} \varphi^{*} \mathcal{R}=\varphi^{*} p^{*} \mathcal{R}$ as $p^{*}$ commutes with direct limits. Therefore, also $p^{*}\left(\varphi^{*}(\psi)\right)=\varphi^{*}\left(p^{*}(\psi)\right)$, as $\varphi^{*}(\psi)$ and $\varphi^{*}\left(p^{*}(\psi)\right)$ restrict to $\psi$ and $p^{*}(\psi)$, respectively, on the homogeneous parts of the Cox sheaves.

The analogous functoriality properties of Cox rings can be deduced from Corollary 3.15 via Corollary 3.6 . 
Remark 3.16. As in Corollary 3.15, let $p: X^{\prime} \rightarrow X$ be a morphism of $k$-varieties, assume that $X^{\prime}$ has a Cox sheaf $\mathcal{R}^{\prime}$ of identity type, and let $\mathcal{R}$ be a Cox sheaf of $X$ of type $\lambda$. Up to twisting $\mathcal{R}^{\prime}$ by an element of $H_{e ́ t}^{1}(k, \widehat{M})$ as in Proposition 3.7 we can assume that $p^{*} \mathcal{R} \cong\left(p^{*} \circ \lambda\right)^{*} \mathcal{R}^{\prime}$. Then there exists a natural morphism of graded $\mathcal{O}_{X^{\prime}}$-algebras $p^{*} \mathcal{R} \rightarrow \mathcal{R}^{\prime}$ as in (3.1), and also a morphism of graded $\mathcal{O}_{X^{-}}$ algebras $\mathcal{R} \rightarrow p_{*} \mathcal{R}^{\prime}$, as $p^{*}$ and $p_{*}$ are adjoint functors. This gives a new proof of the existence statement in [BH03, Proposition 5.3] by considering $k=\bar{k}$ and $\lambda=\operatorname{id}_{\operatorname{Pic}(X)}$. We observe that in general $p_{*} \mathcal{R}^{\prime}$ is not a Cox sheaf of $X$.

Now we turn to existence criteria for Cox rings and Cox sheaves of varieties over nonclosed fields. We start with an example that shows that Cox rings of identity type do not always exist. We also observe that a Cox ring of $X$ of type $\lambda$ exists if $\lambda$ factors through $\operatorname{Pic}(X) \hookrightarrow \operatorname{Pic}\left(X_{\bar{k}}\right)$, as Construction 2.7 can be performed over $k$.

Example 3.17. Let $X \subset \mathbb{P}_{\mathbb{R}}^{2}$ be the conic defined by $x^{2}+y^{2}+z^{2}=0$, with $X(\mathbb{R})=\emptyset$. The base change $X_{\mathbb{C}}$ is the image of the closed immersion

$$
\psi: \mathbb{P}_{\mathbb{C}}^{1} \rightarrow \mathbb{P}_{\mathbb{C}}^{2}, \quad(u: v) \mapsto\left(2 u v: u^{2}-v^{2}: \mathrm{i}\left(u^{2}+v^{2}\right)\right) .
$$

A Cox ring of $\mathbb{P}_{\mathbb{C}}^{1}$ of type $\operatorname{id}_{\operatorname{Pic}\left(\mathbb{P}_{\mathbb{C}}^{1}\right)}$ is $\mathbb{C}[u, v]$, where $u, v \in H^{0}\left(\mathbb{P}_{\mathbb{C}}^{1}, \mathcal{O}(1)\right)$ vanish in $(0: 1),(1: 0)$, respectively. If $X$ has a Cox $\operatorname{ring} R$ of type $\operatorname{id}_{\operatorname{Pic}\left(X_{\mathbb{C}}\right)}$ over $\mathbb{R}$, then $R_{\mathbb{C}}$ is endowed with a natural action of $\mathfrak{g}:=\operatorname{Gal}(\mathbb{C} / \mathbb{R})=\{\mathrm{id}, \mathrm{g}\}$. Via the isomorphism $\psi$, this action pulls back to an action of $\mathfrak{g}$ on the Cox ring $\mathbb{C}[u, v]$ of $\mathbb{P}_{\mathbb{C}}^{1}$ with the following properties. There exists $\alpha \in \mathbb{C}^{\times}$such that $\mathrm{g}(u)=\alpha v$ since g exchanges $(0: 1: \mathrm{i})=\psi((1: 0))$ and $(0: 1:-\mathrm{i})=\psi((0: 1))$. Then $\mathrm{g}^{2}(u)=u$ gives $\mathrm{g}(v)=\mathrm{g}(\alpha)^{-1} u$. Furthermore, g exchanges the points $(1: 0: \mathrm{i})=\psi((1: 1))$ and $(1: 0:-\mathrm{i})=\psi((1:-1))$, hence $\mathrm{g}(u-v)=\alpha v-\mathrm{g}(\alpha)^{-1} u$ should be a scalar multiple of $u+v$. This implies $\alpha=-\mathrm{g}(\alpha)^{-1}$, which is impossible for $\alpha \in \mathbb{C}^{\times}$. Therefore, the conic $X$ without $\mathbb{R}$-rational points does not have a Cox ring of type $\operatorname{id} \operatorname{Pic}\left(X_{\mathbb{C}}\right)$.

Now we compute a Cox ring of $X$ of type $\operatorname{Pic}(X) \hookrightarrow \operatorname{Pic}\left(X_{\mathbb{C}}\right)$. Since $\operatorname{Pic}(X)$ is the subgroup of index 2 of $\operatorname{Pic}\left(X_{\mathbb{C}}\right)$, by Definition 3.12 a Cox ring of $X_{\mathbb{C}}$ of type $\operatorname{Pic}(X) \hookrightarrow \operatorname{Pic}\left(X_{\mathbb{C}}\right)$ is isomorphic via $\psi$ to the subring of $\mathbb{C}[u, v]$ generated by $u^{2}, u v, v^{2}$. Since $\mathbb{C}\left[u^{2}, u v, v^{2}\right]=\mathbb{C}\left[2 u v, u^{2}-v^{2}, \mathrm{i}\left(u^{2}+v^{2}\right)\right]$ as subrings of $\mathbb{C}[u, v]$, we see that the homogeneous coordinate ring $\mathbb{C}[x, y, z] /\left(x^{2}+y^{2}+z^{2}\right)$ of $X_{\mathbb{C}}$ is a $\mathfrak{g}$-equivariant Cox ring of $X_{\mathbb{C}}$ of type $\operatorname{Pic}(X) \hookrightarrow \operatorname{Pic}\left(X_{\mathbb{C}}\right)$. Hence $\mathbb{R}[x, y, z] /\left(x^{2}+\right.$ $\left.y^{2}+z^{2}\right)$ is a Cox ring of $X$ of type $\operatorname{Pic}(X) \hookrightarrow \operatorname{Pic}\left(X_{\mathbb{C}}\right)$ in the sense of Definition 3.3 .

Remark 3.18. Assume that $X$ has a Cox sheaf (ring) of injective type $M^{\prime} \hookrightarrow$ $\operatorname{Pic}\left(X_{\bar{k}}\right)$, where $M^{\prime}$ is a $\mathfrak{g}$-invariant subgroup of $\operatorname{Pic}\left(X_{\bar{k}}\right)$. If $\lambda: M \rightarrow \operatorname{Pic}\left(X_{\bar{k}}\right)$ factors through $M^{\prime}$, Cox sheaves (rings) of $X$ of type $\lambda$ exist by pullback (see Definition 3.12). Therefore, if $X$ has a Cox sheaf (ring) of identity type, then Cox sheaves (rings) of $X$ of all types exist (cf. Sko01, p. 25]).

The next proposition relates the existence of Cox sheaves to the existence of $\mathfrak{g}$-equivariant characters in Construction 2.7 and shows that our definition of Cox sheaves of identity type over nonclosed fields recovers ADHL15, Construction 6.1.3.3] for locally factorial $X$.

Proposition 3.19. Let $\mathcal{M} \subseteq M$ be a finite $\mathfrak{g}$-invariant set of generators for $M$, and $\mathcal{D}$ a finite $\mathfrak{g}$-invariant set of Cartier divisors such that $\lambda(\mathcal{M})=\{[D]: D \in \mathcal{D}\}$. Let

$$
\mathcal{M}_{\lambda}:=\{(m, D) \in \mathcal{M} \times \mathcal{D}:[D]=\lambda(m)\}
$$

with the componentwise action of $\mathfrak{g}$, and let $\Lambda:=\bigoplus_{L \in \mathcal{M}_{\lambda}} \mathbb{Z} L$ with the induced $\mathfrak{g}$-action. Let $\varphi: \Lambda \rightarrow M$ be defined by $\varphi((m, D)):=m$ for all $(m, D) \in \Lambda$, and $\mathcal{S}:=\bigoplus_{(m, D) \in \Lambda} \mathcal{O}_{X_{\bar{k}}}(D)$ the associated $\mathcal{O}_{X_{\bar{k}}}$-algebra as in Construction 2.7. Then 
a Cox sheaf of $X$ of type $\lambda$ exists if and only if there is a $\mathfrak{g}$-equivariant character associated with $\mathcal{S}$.

Proof. Let $\Lambda_{0}$ be the kernel of $\varphi$, and let $\mathcal{S}_{(m, D)}:=\mathcal{O}_{X_{\bar{k}}}(D)$ for all $(m, D) \in \Lambda$. Given a g-equivariant character $\chi: \Lambda_{0} \rightarrow \bar{k}\left(X_{\bar{k}}\right)^{\times}$associated with $\mathcal{S}$, the sheaf $\mathcal{I}$ of ideals of $\mathcal{S}$ defined by $\chi$ is invariant under the action of $\mathfrak{g}$ on $\mathcal{S}$ induced by the natural $\mathfrak{g}$-action on $\bar{k}\left(X_{\bar{k}}\right)$. Therefore, $\mathcal{R}:=\mathcal{S} / \mathcal{I}$ is a $\mathfrak{g}$-equivariant Cox sheaf of $X_{\bar{k}}$ of type $\lambda$, and the sheaf of invariants $\mathcal{R}^{\mathfrak{g}}$ is a Cox sheaf of $X$ of type $\lambda$.

Assume now that there is a Cox sheaf $\mathcal{R}$ of $X$ of type $\lambda$. Then $\mathcal{R}_{\bar{k}}$ is a $\mathfrak{g}$ equivariant Cox sheaf of $X_{\bar{k}}$ of type $\lambda$. By Proposition [2.9, we can assume that $\mathcal{R}_{\bar{k}}=\mathcal{S} / \mathcal{I}$, where $\mathcal{I}$ is the sheaf of ideals of $\mathcal{S}$ defined by a character $\chi: \Lambda_{0} \rightarrow$ $\bar{k}\left(X_{\bar{k}}\right)^{\times}$associated with $\mathcal{S}$. Let $\pi: \mathcal{S} \rightarrow \mathcal{R}_{\bar{k}}$ be the projection.

Let $\left\{\phi_{m, D}\right\}_{(m, D) \in M_{\lambda}}$ be a family of isomorphisms associated with $\mathcal{R}_{\bar{k}}$. Without loss of generality, we can assume that $\phi_{m, D}=\left.\pi\right|_{\mathcal{S}_{(m, D)}} ^{-1}$ for all $(m, D) \in \Lambda$. By Definition 3.1 and Lemma 2.4, for every $g \in \mathfrak{g}$ and every $(m, D) \in \Lambda$, there exists a constant $\alpha_{\mathrm{g},(m, D)} \in \bar{k}^{\times}$such that

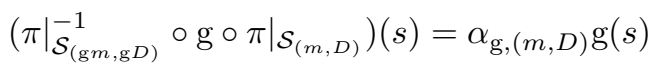

for all sections $s$ of $\mathcal{S}_{(m, D)}=\mathcal{O}_{X_{\bar{k}}}(D)$. These constants satisfy

$$
\alpha_{\mathrm{g}, L+L^{\prime}}=\alpha_{\mathrm{g}, L} \alpha_{\mathrm{g}, L^{\prime}} \quad \alpha_{\mathrm{gg}^{\prime}, L}=\alpha_{\mathrm{g}, \mathrm{g}^{\prime}(L)} \mathrm{g}\left(\alpha_{\mathrm{g}^{\prime}, L}\right)
$$

for all $L, L^{\prime} \in \Lambda$ and all $\mathrm{g}, \mathrm{g}^{\prime} \in \mathfrak{g}$. Moreover, $\chi(\mathrm{g}(E))=\alpha_{\mathrm{g},-E \mathrm{~g}}(\chi(E))$ for all $E \in \Lambda_{0}$.

Consider $\left(\bar{k}^{\times}\right)^{\mathcal{M}_{\lambda}}$ with the induced $\mathfrak{g}$-action, that is,

$$
\mathrm{g} *\left(\beta_{L}\right)_{L \in \mathcal{M}_{\lambda}}:=\left(\mathrm{g}\left(\beta_{\mathrm{g}^{-1}(L)}\right)\right)_{L \in \mathcal{M}_{\lambda}}
$$

for all $\mathrm{g} \in \mathfrak{g}$ and $\left(\beta_{L}\right)_{L \in \mathcal{M}_{\lambda}} \in\left(\bar{k}^{\times}\right)^{\mathcal{M}_{\lambda}}$. The map $\alpha: \mathfrak{g} \rightarrow\left(\bar{k}^{\times}\right)^{\mathcal{M}_{\lambda}}$ defined by $\alpha(\mathrm{g}):=\left(\alpha_{\mathrm{g}, \mathrm{g}^{-1}(L)}\right)_{L \in \mathcal{M}_{\lambda}}$ is a cocycle. By [Gro03, $\S \mathrm{V} .7$, Proposition V.8.1], there is an equivalence of categories between the category of finite sets with a continuous $\mathfrak{g}$-action and the category of finite étale $k$-algebras. Let $K$ be a finite étale $k$-algebra corresponding to $\mathcal{M}_{\lambda}$. Then $\left(\bar{k}^{\times}\right)^{\mathcal{M}_{\lambda}}$ with the $\mathfrak{g}$-action recalled above is the set of $\bar{k}$-rational points of the Weil restriction $\operatorname{Res}_{K / k} \mathbb{G}_{m}$. By Hilbert's Theorem 90 and the Lemma of Eckmann-Faddeev-Shapiro [KMRT98, Theorem 29.2 and Lemma 29.6],

$$
H^{1}\left(\mathfrak{g},\left(\bar{k}^{\times}\right)^{\mathcal{M}_{\lambda}}\right) \cong H_{e ́ t}^{1}\left(k, \operatorname{Res}_{K / k} \mathbb{G}_{m}\right) \cong H_{e ́ t}^{1}\left(K, \mathbb{G}_{m}\right)=0 .
$$

Therefore, there is $\beta=\left(\beta_{L}\right)_{L \in \mathcal{M}_{\lambda}} \in \mathcal{M}_{\lambda} \times \bar{k}^{\times}$such that $\alpha(\mathrm{g})=\beta^{-1} \mathrm{~g} * \beta$ for all $\mathrm{g} \in \mathfrak{g}$. That is, $\alpha_{\mathrm{g}, L}=\beta_{\mathrm{g}(L)}^{-1} \mathrm{~g}\left(\beta_{L}\right)$ for all $\mathrm{g} \in \mathfrak{g}$ and $L \in \mathcal{M}_{\lambda}$. Given $L^{\prime} \in \Lambda$, write $L^{\prime}=\sum_{L \in \mathcal{M}_{\lambda}} a_{L} L$ with $a_{L} \in \mathbb{Z}$, and define

$$
\beta_{L^{\prime}}:=\prod_{L \in \mathcal{M}_{\lambda}} \beta_{L}^{a_{L}} .
$$

The homomorphism $\chi^{\prime}: \Lambda_{0} \rightarrow \bar{k}\left(X_{\bar{k}}\right)^{\times}$that sends $E \in \Lambda_{0}$ to $\beta_{E}^{-1} \chi(E)$ is $\mathfrak{g}$ equivariant and satisfies $\operatorname{div}_{0}\left(\chi^{\prime}((0, D))\right)=D$ for all $(0, D) \in \Lambda_{0}$.

From [CTS87, Proposition 2.2.8], we know that the existence of universal torsors (that is, torsors of type $\left.\mathrm{id}_{\operatorname{Pic}\left(X_{\bar{k}}\right)}\right)$ for a smooth variety $X$ over a nonclosed field $k$ is equivalent to the existence of a $\mathfrak{g}$-equivariant splitting of the exact sequence

$$
1 \rightarrow \bar{k}^{\times} \rightarrow \bar{k}\left(X_{\bar{k}}\right)^{\times} \rightarrow \bar{k}\left(X_{\bar{k}}\right)^{\times} / \bar{k}^{\times} \rightarrow 1 .
$$

Moreover, if $U$ is a nonempty open subset of $X$, and $M$ is the kernel of the natural homomorphism $\operatorname{Pic}\left(X_{\bar{k}}\right) \rightarrow \operatorname{Pic}\left(U_{\bar{k}}\right)$, the existence of torsors of injective type 
$M \hookrightarrow \operatorname{Pic}\left(X_{\bar{k}}\right)$ is equivalent to the existence of a $\mathfrak{g}$-equivariant splitting of the exact sequence

$$
1 \rightarrow \bar{k}^{\times} \rightarrow \bar{k}\left[U_{\bar{k}}\right]^{\times} \rightarrow \bar{k}\left[U_{\bar{k}}\right]^{\times} / \bar{k}^{\times} \rightarrow 1 .
$$

As a consequence of Corollaries 3.6 and 3.11, this is also equivalent to the existence of Cox sheaves (and Cox rings if $M=M_{\mathrm{eff}}$ ) of $X$ of the same type.

In our more general setting, where $X$ can be singular, we show how to construct a Cox sheaf of $X$ of type $\lambda$ starting from $\mathfrak{g}$-equivariant splittings of such exact sequences. Then Proposition 3.9 gives a torsor of $X$ of type $\lambda$ in this setting, generalizing the results of [CTS87] mentioned above.

Construction 3.20. Let $U \subseteq X$ be a nonempty open subset such that $\lambda(M)$ is contained in the kernel of the natural morphism $\operatorname{Pic}\left(X_{\bar{k}}\right) \rightarrow \operatorname{Pic}\left(U_{\bar{k}}\right)$. Assume that (3.3) admits a $\mathfrak{g}$-equivariant splitting $\sigma: \bar{k}\left[U_{\bar{k}}\right]^{\times} \rightarrow \bar{k}^{\times}$. For every $m \in M$, let $D_{m}$ be a Cartier divisor supported on $X_{\bar{k}} \backslash U_{\bar{k}}$ representing the class $\lambda(m)$ in $\operatorname{Pic}\left(X_{\bar{k}}\right)$, and let $\mathcal{R}_{m}:=\mathcal{O}_{X}\left(D_{m}\right)$. Endow $\mathcal{R}:=\bigoplus_{m \in M} \mathcal{R}_{m}$ with the following multiplication of sections induced by $\sigma$. For every open subset $V$ of $X_{\bar{k}}$ and homogeneous sections $s_{1}, s_{2} \in \mathcal{R}(V)$ of degree $m_{1}, m_{2} \in M$, respectively, let $f \in \bar{k}\left[U_{\bar{k}}\right]^{\times}$be the unique element such that $D_{m_{1}}+D_{m_{2}}=\operatorname{div}_{D_{m_{1}+m_{2}}}(f)$ and $\sigma(f)=1$. Define $s_{1} s_{2}:=$ $f s_{1} s_{2} \in \mathcal{R}_{m_{1}+m_{2}}(V)$, where the product on the right is computed in $\bar{k}\left(X_{\bar{k}}\right)$. Then $\mathcal{R}$ is a Cox sheaf of $X_{\bar{k}}$ of type $\lambda$ that is equivariant with respect to the following action of $\mathfrak{g}$. For every $\mathrm{g} \in \mathfrak{g}$ and $m \in M$, let $f \in \bar{k}\left(X_{\bar{k}}\right)^{\times}$be the unique element such that $\mathrm{g} D_{m}=\operatorname{div}_{D_{\mathrm{g} m}}(f)$ and $\sigma(f)=1$. Define $\mathrm{g} * s:=f \mathrm{~g}(s) \in \mathcal{R}_{\mathrm{g} m}$ for all sections $s \in \mathcal{R}_{m}$, where the product on the right is computed in $\bar{k}\left(X_{\bar{k}}\right)$.

Then the sheaf of invariants $\mathcal{R}^{\mathfrak{g}}$ is a Cox sheaf of $X$ of type $\lambda$ by Proposition 3.4. and $\mathcal{R}^{\mathfrak{g}}(X)$ is a Cox ring of $X$ of type $\lambda$ by Corollary [3.6.

Remark 3.21. We note that a $\mathfrak{g}$-equivariant splitting of (3.2) induces a $\mathfrak{g}$-equivariant splitting of (3.3) for all nonempty open subsets $U$ of $X$. Hence a $\mathfrak{g}$-equivariant splitting of (3.2) ensures the existence of Cox rings and Cox sheaves of $X$ of every type.

The following proposition generalizes [CTS87, Proposition 2.2.8(v)].

Proposition 3.22. If $X_{\bar{k}}$ is locally factorial, and $X$ has a Cox sheaf of injective type $\lambda$, then the natural exact sequence (3.3) has a $\mathfrak{g}$-equivariant splitting for every nonempty open subset $U \subseteq X$ such that $\lambda(M)$ is the kernel of the natural morphism $\operatorname{Pic}\left(X_{\bar{k}}\right) \rightarrow \operatorname{Pic}\left(U_{\bar{k}}\right)$.

If in addition $k$ is perfect and $\lambda=\mathrm{id}_{\mathrm{Pic}\left(X_{\bar{k}}\right)}$, also the exact sequence (3.2) admits a $\mathfrak{g}$-equivariant splitting.

Proof. Let $U \subseteq X$ be as in the statement. Then the group $\Lambda$ of Cartier divisors on $X_{\bar{k}}$ supported outside $U_{\bar{k}}$ is free, finitely generated and has a $\mathfrak{g}$-invariant basis (consisting of the prime divisors supported outside $U_{\bar{k}}$ ). The group $\bar{k}\left[U_{\bar{k}}\right]^{\times} / \bar{k}^{\times}$is naturally identified with the subgroup $\Lambda_{0} \subseteq \Lambda$ of principal divisors. By Proposition 3.19 there exists a $\mathfrak{g}$-equivariant character $\chi: \Lambda_{0} \rightarrow \bar{k}\left(X_{\bar{k}}\right)^{\times}$associated with $\mathcal{S}:=$ $\bigoplus_{D \in \Lambda} \mathcal{O}_{X}(D)$. Since every element of $\Lambda_{0}$ is supported outside $U_{\bar{k}}$, the image of $\chi$ is contained in $\bar{k}\left[U_{\bar{k}}\right]^{\times}$. Therefore, $\chi$ is a $\mathfrak{g}$-equivariant splitting of the exact sequence (3.3) associated with $U$.

Assume now that $k$ is perfect and that $\lambda=\operatorname{id}_{\operatorname{Pic}\left(X_{\bar{k}}\right)}$. Let $X^{\prime}$ be the smooth locus of $X_{\bar{k}}$ and $U^{\prime}:=U_{\bar{k}} \cap X^{\prime}$. Since $X_{\bar{k}}$ is normal, its singular locus has codimension $\geq 2$. Hence $\bar{k}\left(X_{\bar{k}}\right)=\bar{k}\left(X^{\prime}\right)$ and $\bar{k}\left[U_{\bar{k}}\right]=\bar{k}\left[U^{\prime}\right]$. Then, according to [CTS87, Proposition 2.2.8], the exact sequence (3.2) admits a $\mathfrak{g}$-equivariant splitting whenever (3.3) does. 
Every rational point $x \in U(k)$ defines a g-equivariant splitting $\sigma_{x}: \bar{k}\left[U_{\bar{k}}\right]^{\times} \rightarrow \bar{k}^{\times}$ of (3.3) by $\sigma_{x}(f):=f(x)$ for all $f \in \bar{k}\left[U_{\bar{k}}\right]^{\times}$. Moreover, every smooth $k$-rational point on $X$ defines a $\mathfrak{g}$-equivariant splitting of (3.2) by [CTS87, Remarque 2.2.3]. Therefore, if $X$ has a smooth $k$-rational point, Cox sheaves and Cox rings of $X$ of every type exist by Construction 3.20 . If $X(k) \neq \emptyset$, the same result can be obtained combining CTS77, Proposition 1] with Proposition 3.10.

The following construction generalizes [ADHL15, Construction 1.4.2.3].

Construction 3.23. Let $x \in X(k)$. Let $\mathcal{M}, \mathcal{D}, \mathcal{M}_{\lambda}, \Lambda, \varphi, \mathcal{S}$ be as in Proposition 3.19, Let $\Lambda_{0}$ be the kernel of $\varphi$. The morphism $\chi: \Lambda_{0} \rightarrow \bar{k}\left(X_{\bar{k}}\right)^{\times}$that sends $(0, D) \in \Lambda_{0}$ to the unique element $f \in \bar{k}\left(X_{\bar{k}}\right)^{\times}$such that $\operatorname{div}_{0}(f)=D$ and $f(x)=1$ is a $\mathfrak{g}$-equivariant character associated with $\mathcal{S}$. Let $\mathcal{I}$ be the sheaf of ideals of $\mathcal{S}$ defined by $\chi$ as in Construction 2.7. Then the Cox sheaf $\mathcal{R}:=\mathcal{S} / \mathcal{I}$ is $\mathfrak{g}$-equivariant by Proposition 3.19. Let $\pi: \operatorname{Spec}_{X} \mathcal{R}^{\mathfrak{g}} \rightarrow X$ be the induced torsor of type $\lambda$. Then $x \in \pi\left(\left(\operatorname{Spec}_{X} \mathcal{R}^{\mathfrak{g}}\right)(k)\right)$.

Proof. The set of Cartier divisors of $X_{\bar{k}}$ that do not contain $x$ in their support form a $\mathfrak{g}$-invariant group that generates $\operatorname{Pic}\left(X_{\bar{k}}\right)$. Indeed, if $D=\left\{\left(U_{i}, f_{i}\right)\right\}_{i}$ is a Cartier divisor on $X_{\bar{k}}$ that contains $x$ in its support, take $j$ such that $x \in U_{j}$. Then the $\operatorname{divisor} \operatorname{div}_{D}\left(f_{j}^{-1}\right)$ is linearly equivalent to $D$ and is supported outside $x$. Therefore, it is always possible to choose a set $\mathcal{D}$ as in the statement. The character $\chi$ defined by $x$ as above is $\mathfrak{g}$-equivariant because $x$ is $\mathfrak{g}$-invariant.

Let $U$ be an affine open neighborhood of $x$ in $X$. Then the homomorphism $\psi: \mathcal{S}\left(U_{\bar{k}}\right) \rightarrow \bar{k}$ defined by $\psi(s):=s(x)$ for all homogeneous sections $s \in \mathcal{S}\left(U_{\bar{k}}\right)$ is well-defined because $D$ is supported outside $x$ for all $(m, D) \in \Lambda$, and $\mathfrak{g}$-equivariant because $x$ is $\mathfrak{g}$-invariant. Since $\chi(E)(x)=1$ for all $E \in \Lambda_{0}$, the homomorphism $\psi$ factors through $\mathcal{R}\left(U_{\bar{k}}\right)$ and defines a $k$-rational point on $\pi^{-1}(x)$ by Galois descent.

Remark 3.24. If $X$ is a smooth complete toric variety containing an open torus $U$, then we can apply Construction 3.23 with $M:=\operatorname{Pic}\left(X_{\bar{k}}\right), \lambda:=\operatorname{id}_{\operatorname{Pic}\left(X_{\bar{k}}\right)}, x$ the neutral element of $U, \mathcal{D}$ the set of $U$-invariant divisors on $X_{\bar{k}}$, and $\mathcal{M}$ the set of their classes. The resulting Cox sheaf corresponds to the principal universal torsor described in [CTS87, §2.4.4] and [Sal98, end of $\S 8]$.

We recall that if $\lambda$ factors through $\operatorname{Pic}(X) \hookrightarrow \operatorname{Pic}\left(X_{\bar{k}}\right)$, a Cox ring of $X$ of type $\lambda$ always exists, even if $X(k)=\emptyset$. The following example shows that the existence of a $k$-rational point on $X$ is not necessary for the existence of Cox rings of $X$ of arbitrary type. See CTS87, Exemples 2.2.12] for further examples.

Example 3.25. Let $k$ be an arbitrary number field. Let $X$ be the smooth projective fourfold over $k$ in [Sme17, Theorem 3.5]. Then $X(k)=\emptyset$, and $X$ is a counterexample to the Hasse principle, but there is no étale (and hence no algebraic) Brauer-Manin obstruction to the Hasse principle. Moreover, $\operatorname{Pic}\left(X_{\bar{k}}\right)$ is a finitely generated abelian group, as the Albanese variety of $X$ is trivial. Therefore, $X$ has a universal torsor by [Sko01, Corollary 6.1.3], and Cox rings and Cox sheaves of $X$ of all types exist by Proposition 3.10 and Remark 3.18.

Proof of Theorem 1.2. For the statements regarding Cox sheaves, see Proposition 3.19. Construction 3.20. Remark 3.21. Proposition 3.22 and Construction 3.23. For the statements regarding torsors and Cox rings, we combine these with Theorem 1.1

\section{Finitely generated Cox Rings}

We observe that, for every Cox sheaf $\mathcal{R}$ of $X$ of type $\lambda$, the natural morphism $\operatorname{Spec}_{X} \mathcal{R} \rightarrow \operatorname{Spec} \mathcal{R}(X)$ is equivariant under the $\widehat{M}$-actions on $\operatorname{Spec}_{X} \mathcal{R}$ and 
Spec $\mathcal{R}(X)$ induced by the $M$-grading on $\mathcal{R}$ and $\mathcal{R}(X)$, respectively. We show that, if $\mathcal{R}(X)$ is finitely generated as a $k$-algebra, this morphism can be used to realize the torsor $\operatorname{Spec}_{X} \mathcal{R}$ as a quasiaffine variety, as in ADHL15, Construction 1.6.3.1] and [BH03, Proposition 3.10].

Proposition 4.1. Assume that $k=\bar{k}$, that $\mathcal{R}$ is a Cox sheaf of $X$ of type $\lambda$ such that $\mathcal{R}(X)$ is finitely generated as a $k$-algebra, and that there are nonzero homogeneous sections $f_{1}, \ldots, f_{t} \in \mathcal{R}(X)$ of degrees $m_{1}, \ldots, m_{t} \in M$, respectively, such that the open subsets $X \backslash \operatorname{Supp}\left(\operatorname{div}_{D_{i}}\left(\phi_{m_{i}, D_{i}}\left(f_{i}\right)\right)\right)$ are affine and cover $X$.

Then the natural morphism $\operatorname{Spec}_{X} \mathcal{R} \rightarrow \operatorname{Spec} \mathcal{R}(X)$ is an $\widehat{M}$-equivariant open immersion, and the complement of the image is defined by the ideal $\sqrt{\left(f_{1}, \ldots, f_{t}\right)}$ of $\mathcal{R}(X)$.

Proof. Let $\pi: \operatorname{Spec}_{X} \mathcal{R} \rightarrow X$ be the morphism induced by $\mathcal{O}_{X} \subseteq \mathcal{R}$. The open subsets $\pi^{-1}\left(X \backslash \operatorname{Supp}\left(\operatorname{div}_{D_{i}}\left(\phi_{m_{i}, D_{i}}\left(f_{i}\right)\right)\right)\right.$ are affine and cover $\operatorname{Spec}_{X} \mathcal{R}$. Moreover,

$$
\mathcal{R}\left(X \backslash \operatorname{Supp}\left(\operatorname{div}_{D_{i}}\left(\phi_{m_{i}, D_{i}}\left(f_{i}\right)\right)\right)=\mathcal{R}(X)\left[f_{i}^{-1}\right]\right.
$$

for all $i \in\{1, \ldots, t\}$. Hence $\operatorname{Spec}_{X} \mathcal{R} \rightarrow \operatorname{Spec} \mathcal{R}(X)$ is an open immersion whose image is the union of the principal open subsets of $\operatorname{Spec} \mathcal{R}(X)$ defined by $f_{i}$ for $i \in\{1, \ldots, t\}$.

Remark 4.2. The assumption of Proposition 4.1 on the affine open covering is equivalent to the requirement that there are effective Cartier divisors $D_{1}, \ldots, D_{t}$ on $X$ such that $\left[D_{i}\right] \in \lambda(M)$ for all $i \in\{1, \ldots, t\}$ and such that the open subsets $X \backslash \operatorname{Supp}\left(D_{i}\right)$ are affine and cover $X$. If $\lambda(M)=\operatorname{Pic}(X)$, this is the definition of divisorial variety [Bor63, §3]. Among those there are quasi-projective varieties and locally factorial varieties Bor63, §4].

If $\lambda(M)$ contains the class of an ample invertible sheaf on $X$, then the hypothesis of Proposition 4.1 on the affine open covering is satisfied, and the open immersion $\operatorname{Spec}_{X} \mathcal{R} \rightarrow \operatorname{Spec} \mathcal{R}(X)$ can be characterized as follows (cf. ADHL15, Corollary 1.6.3.6]).

Corollary 4.3. Assume that $k=\bar{k}$, that $X$ is projective and has a Cox sheaf $\mathcal{R}$ of type $\lambda$ such that $\mathcal{R}(X)$ is a finitely generated $k$-algebra, and there is $m \in M$ such that $\lambda(m)$ is very ample. Then $\operatorname{Spec}_{X} \mathcal{R} \rightarrow \operatorname{Spec} \mathcal{R}(X)$ is a $\widehat{M}$-equivariant open immersion and the complement of the image is defined by the ideal $\sqrt{\left\langle\mathcal{R}(X)_{m}\right\rangle}$ of $\mathcal{R}(X)$, where $\left\langle\mathcal{R}(X)_{m}\right\rangle$ is the ideal generated by the degree-m-part of $\mathcal{R}(X)$.

If the Cox sheaf is defined over a nonclosed field $k$, the open immersions in Proposition 4.1 and Corollary 4.3 are $\mathfrak{g}$-equivariant, and hence defined over $k$.

Now we explain how to realize a finitely generated Cox ring as a quotient of a polynomial ring. Without loss of generality, we may assume that $M=M_{\text {eff }}$ (cf. Remark 2.13).

Proposition 4.4. Assume that $k=\bar{k}$. Let $m_{1}, \ldots, m_{N} \in M$ be generators of $M_{\text {eff }}$. Let $\Lambda:=\bigoplus_{i=1}^{N} \mathbb{Z} m_{i}$, and let $\Lambda_{0}$ be the kernel of the natural homomorphism $\varphi: \Lambda \rightarrow$ $M$. For $i \in\{1, \ldots, N\}$, let $D_{i}$ be a Cartier divisor representing the class $\lambda\left(m_{i}\right)$ in $\operatorname{Pic}(X)$, and for every $L=\sum_{i=1}^{N} a_{i} m_{i}$ of $\Lambda$, let $D_{L}:=\sum_{i=1}^{N} a_{i} D_{i}$. Let $\chi: \Lambda_{0} \rightarrow$ $k(X)^{\times}$be a character associated with the $\mathcal{O}_{X}$-algebra $\mathcal{S}:=\bigoplus_{L \in \Lambda} \mathcal{O}_{X}\left(D_{L}\right)$.

Endow $S:=k\left[\eta_{1}, \ldots, \eta_{N}\right]$ with the $M$-grading induced by assigning degree $m_{i}$ to $\eta_{i}$ for each $i \in\{1, \ldots, N\}$. For every $L \in \Lambda$, let

$$
\phi_{L}: S_{\varphi(L)} \rightarrow H^{0}\left(X, \mathcal{O}_{X}\left(D_{L}\right)\right)
$$

be the linear map that sends $\eta_{1}^{a_{1}} \cdots \eta_{N}^{a_{N}}$ to $\chi\left(\sum_{i=1}^{N} a_{i} m_{i}-L\right)$ for all nonnegative integers $a_{1}, \ldots, a_{N}$ such that $\sum_{i=1}^{N} a_{i} m_{i}=\varphi(L)$ in $M$. Let $g_{1}, \ldots, g_{s} \in S$ be 
homogeneous elements such that, for each $i \in\{1, \ldots, s\}$, if $g_{i}$ has degree $\varphi\left(L_{i}\right)$ with $L_{i} \in \Lambda$, then $\phi_{L_{i}}\left(g_{i}\right)=0$.

Then $R:=S /\left(g_{1}, \ldots, g_{s}\right)$ is a Cox ring of $X$ of type $\lambda$ if and only if the linear map

$$
\phi_{\varphi(L), D_{L}}: R_{\varphi(L)} \rightarrow H^{0}\left(X, \mathcal{O}_{X}\left(D_{L}\right)\right)
$$

induced by $\phi_{L}$ is an isomorphism for all $L \in \Lambda$.

Conversely, if $R$ is a finitely generated Cox ring of $X$ of type $\lambda$, then there are generators $m_{1}, \ldots, m_{N}$ of $M_{\mathrm{eff}}$, a character $\chi$ and polynomials $g_{1}, \ldots, g_{s} \in$ $k\left[\eta_{1}, \ldots, \eta_{N}\right]$ as above such that $R \cong k\left[\eta_{1}, \ldots, \eta_{N}\right] /\left(g_{1}, \ldots, g_{s}\right)$.

Proof. For the first statement, we notice that, for all $L_{1}, L_{2} \in \Lambda$ and all $s_{1}, s_{2} \in S$ homogeneous of degrees $\varphi\left(L_{1}\right), \varphi\left(L_{2}\right)$, respectively,

$$
\phi_{L_{1}}\left(s_{1}\right) \phi_{L_{2}}\left(s_{2}\right)=\phi_{L_{1}+L_{2}}\left(s_{1} s_{2}\right)
$$

because $\chi$ is a group homomorphism.

Conversely, assume that $R$ is a finitely generated Cox $\operatorname{ring}$ of $X$ of type $\lambda$, and let $s_{1}, \ldots, s_{N}$ be a finite set of homogeneous elements that generate $R$. For every $i \in\{1, \ldots, N\}$, let $m_{i}$ be the degree of $s_{i}$. Sending $\eta_{i} \mapsto s_{i}$ defines a surjective homomorphism $\pi: k\left[\eta_{1}, \ldots, \eta_{N}\right] \rightarrow R$ of $M$-graded rings, where the grading on $S:=k\left[\eta_{1}, \ldots, \eta_{N}\right]$ is defined by assigning degree $m_{i}$ to $\eta_{i}$ for all $i \in\{1, \ldots, N\}$. Since $S$ is noetherian, the kernel of $\pi$ is generated by finitely many homogeneous elements $g_{1}, \ldots, g_{s}$.

Since $s_{1}, \ldots, s_{N}$ generate $R$, the elements $m_{1}, \ldots, m_{N}$ generate $M_{\text {eff }}$. Let $\Lambda:=$ $\bigoplus_{i=1}^{N} \mathbb{Z} m_{i}$, and let $\Lambda_{0}$ be the kernel of the natural homomorphism $\varphi: \Lambda \rightarrow M$. For every $i \in\{1, \ldots, N\}$, let $D_{i}:=\operatorname{div}\left(s_{i}\right)$, and, for every $L=\sum_{i=1}^{N} a_{i} m_{i}$ in $\Lambda$, let $D_{L}:=\sum_{i=1}^{N} a_{i} D_{i}$.

Let $\left\{\phi_{m, D}\right\}_{(m, D) \in M_{\lambda}}$ be a family of isomorphisms associated with $R$ as in Proposition 2.3. Let $\Lambda_{+}$be the monoid generated by $m_{1}, \ldots, m_{N}$. For every $L=$ $\sum_{i=1}^{N} a_{i} m_{i} \in \Lambda_{+}$, let $\alpha_{L}:=\phi_{\varphi(L), D_{L}}\left(s_{1}^{a_{1}} \cdots s_{N}^{a_{N}}\right)$. For every $L \in \Lambda$ such that $\lambda(\varphi(L))$ is an effective class, write $L=L^{+}-L^{-}$with $L^{+}, L^{-} \in \Lambda_{+}$, and define $\alpha_{L}:=$ $\alpha_{L^{+}} \alpha_{L^{-}}^{-1} \alpha$, where $\alpha \in k^{\times}$is the unique constant with $\phi_{\varphi(L), D_{L}}(s) \phi_{\varphi\left(L^{-}\right), D_{L^{-}}}\left(s^{\prime}\right)=$ $\alpha \phi_{\varphi\left(L^{+}\right), D_{L^{+}}}\left(s s^{\prime}\right)$ for all $s \in R_{\varphi(L)}$ and $s^{\prime} \in R_{\varphi\left(L^{-}\right)}$. The constant $\alpha_{L}$ does not depend on the choice of $L^{+}$and $L^{-}$. If $\lambda(\varphi(L))$ is not effective, take $\alpha_{L}:=1$.

For every $L \in \Lambda$, let $\phi_{L}: S_{\varphi(L)} \rightarrow H^{0}\left(X, \mathcal{O}_{X}\left(D_{L}\right)\right)$ be the linear map that sends $\eta_{1}^{a_{1}} \cdots \eta_{N}^{a_{N}}$ to $\alpha_{L}^{-1} \phi_{\varphi(L), D_{L}}\left(s_{1}^{a_{1}} \cdots s_{N}^{a_{N}}\right)$ for all $\left(a_{1}, \ldots, a_{N}\right) \in \mathbb{Z}_{\geq 0}^{N}$ such that $\sum_{i=1}^{N} a_{i} m_{i}=\varphi(L)$ in $M$. The morphisms $\phi_{L}$ satisfy

$$
\phi_{L}(s) \phi_{L^{\prime}}\left(s^{\prime}\right)=\phi_{L+L^{\prime}}\left(s s^{\prime}\right)
$$

for all $s, s^{\prime} \in S$ homogeneous of degrees $\varphi(L), \varphi\left(L^{\prime}\right)$, respectively, for all $L, L^{\prime} \in$ $\Lambda$. Then the map $\chi: \Lambda_{0} \rightarrow k(X)^{\times}$defined by $\chi(E):=\phi_{-E}(1)$, for all $E \in$ $\Lambda_{0}$, is a character associated with $\bigoplus_{L \in \Lambda} \mathcal{O}_{X}\left(D_{L}\right)$ that defines on the $k$-algebra $k\left[\eta_{1}, \ldots, \eta_{N}\right] /\left(g_{1}, \ldots, g_{s}\right)$ a structure of Cox ring of $X$ of type $\lambda$, which coincides with the one induced by $R$ via $\pi$.

Remark 4.5. Let $k$ be an arbitrary field, and consider the construction in Proposition 4.4 for $X_{\bar{k}}$. If the set $\left\{\left(m_{1}, D_{1}\right), \ldots,\left(m_{N}, D_{N}\right)\right\}$ is $\mathfrak{g}$-invariant with respect to the componentwise action of $\mathfrak{g}$ and if the character $\chi$ is $\mathfrak{g}$-equivariant, then $\phi_{\mathrm{g} L} \circ \mathrm{g}=\mathrm{g} \circ \phi_{L}$ for all $\mathrm{g} \in \mathfrak{g}$ and all $L \in \Lambda$, where $\left\{\phi_{L}\right\}_{L \in \Lambda}$ are the morphisms defined by $\chi$ and $\mathfrak{g}$ acts on $S$ by permuting the generators as follows: $\mathrm{g}\left(\eta_{i}\right)=\eta_{j}$ if $\mathrm{g}\left(m_{i}\right)=m_{j}$. Therefore, if $R:=S /\left(g_{1}, \ldots, g_{s}\right)$ is a Cox ring of $X_{\bar{k}}$ of type $\lambda$, it is endowed with a natural $\mathfrak{g}$-action, and descends to a Cox ring $R^{\mathfrak{g}}$ of $X$ of type $\lambda$ by Proposition 3.4. The ring $R^{\mathfrak{g}}$ is a finitely generated $k$-algebra by faithfully flat descent. 
We conclude this section by proving that finite generation is preserved under pullback of Cox rings.

Proposition 4.6. Let $\varphi: M^{\prime} \rightarrow M$ be a homomorphism of finitely generated abelian groups, and $R$ a finitely generated $M$-graded $k$-algebra. Then $\varphi^{*} R:=$ $\bigoplus_{m^{\prime} \in M^{\prime}} R_{\varphi\left(m^{\prime}\right)}$ is finitely generated as a $k$-algebra.

Proof. If $\varphi$ is injective, the statement holds by ADHL15, Proposition 1.1.2.4]. Hence, by factoring $\varphi$ through the inclusion $\varphi\left(M^{\prime}\right) \subseteq M$, we can assume without loss of generality that $\varphi$ is surjective.

Fix homogeneous generators $s_{1}, \ldots, s_{n}$ of $R$ of degrees $m_{1}, \ldots, m_{n} \in M$, respectively, and let $m_{1}^{\prime}, \ldots, m_{n^{\prime}}^{\prime}$ be generators for $M^{\prime}$. Up to enlarging the sets $\left(s_{1}, \ldots, s_{n}\right)$ and $\left(m_{1}^{\prime}, \ldots, m_{n^{\prime}}^{\prime}\right)$ and permuting the indices, we can assume that $n=n^{\prime}$ and that $\varphi\left(m_{i}^{\prime}\right)=m_{i}$ for all $i \in\{1, \ldots n\}$. Let $M_{0}$ be the kernel of $\varphi$, and fix elements $m_{1}^{\prime \prime}, \ldots, m_{r}^{\prime \prime} \in M_{0}$ that generate $M_{0}$ as a monoid. Let

$$
\psi: k\left[t_{1}, \ldots, t_{n}, u_{1}, \ldots, u_{r}\right] \rightarrow \bigoplus_{m^{\prime} \in M^{\prime}} R_{\varphi\left(m^{\prime}\right)}=: \varphi^{*} R
$$

be the morphism that sends the variable $t_{i}$ to the section $s_{i}$ in degree $m_{i}^{\prime}$, for all $i \in$ $\{1, \ldots, n\}$, and the variable $u_{j}$ to the section 1 in degree $m_{j}^{\prime \prime}$, for all $j \in\{1, \ldots, r\}$.

We show that $\psi$ is surjective. Let $s$ be a homogeneous element of $\varphi^{*} R$ of arbitrary degree $m^{\prime} \in M^{\prime}$. Since $s \in R_{\varphi\left(m^{\prime}\right)}$ and $R$ is generated by $s_{1}, \ldots, s_{n}$ as a $k$-algebra, we can assume, without loss of generality, that $s$ is a monomial in the generators, i.e., $s=\prod_{i=1}^{n} s_{i}^{a_{i}}$ for suitable nonnegative integers $a_{1}, \ldots, a_{n}$. Then the element $m^{\prime \prime}:=m^{\prime}-\sum_{i=1}^{n} a_{i} m_{i}^{\prime}$ is in $M_{0}$. Write $m^{\prime \prime}=\sum_{i=1}^{r} b_{i} m_{i}^{\prime \prime}$ for suitable nonnegative integers $b_{1}, \ldots, b_{r}$. Then $s=\psi\left(\left(\prod_{i=1}^{n} t_{i}^{a_{i}}\right)\left(\prod_{j=1}^{r} u_{j}^{b_{j}}\right)\right)$.

The pullback of a finitely generated $k$-algebra under an injective morphism of grading groups is also called a Veronese subalgebra; see ADHL15. for example. Generators and relations of Veronese subalgebras can be computed via an algorithm provided by the Maple package for Mori dream spaces developed by Hausen and Keicher HK15.

\section{Arithmetic applications}

The embedding from Proposition 4.1 provides an explicit description of torsors as open subsets of closed subsets of affine spaces. Such a description of universal torsors has been used to prove Manin's conjecture on the distribution of rational points for certain varieties over number fields; see [Sal98, Bre02, BBD07, BBS14, FP16, for example. In these cases, the varieties are split (see Section 1.1).

If a variety is not split, other torsors may give more suitable parameterizations for the purpose of counting rational points. We present three examples where the parameterizations for the study of rational points on nonsplit varieties induced by torsors of non-identity type can be determined by computing the associated Cox rings.

Example 5.1. See BB07] for a proof of Manin's conjecture for the nonsplit singular quartic del Pezzo surface $S \subset \mathbb{P}_{\mathbb{Q}}^{4}$ defined by

$$
x_{0} x_{1}-x_{2}^{2}=x_{0}^{2}-x_{1} x_{4}+x_{3}^{2}=0 .
$$

Over $\mathbb{Q}(\mathrm{i})$, it contains exactly one singular point of type $\mathbf{D}_{4}$ in $(0: 0: 0: 0: 1)$ and two conjugate lines. Let $X$ be a minimal desingularization of $S$. A central step in this proof of Manin's conjecture is the parameterization of $X(\mathbb{Q})$ in BB07, $\S 3]$, which is obtained by a sequence of elementary manipulations of the defining equations. 
One can show that this parameterization of $X(\mathbb{Q})$ is induced by a $\mathbb{Z}$-model of a torsor of type $\operatorname{Pic}(X) \hookrightarrow \operatorname{Pic}\left(X_{\bar{k}}\right)$. To prove it, one can start with the Cox ring of $X_{\overline{\mathbb{Q}}}$ of identity type computed in [Der14, §3.4] and use the theory developed in our present work together with the MDS package [HK15] and results on models of torsors from [Pie15, §1, §3].

Example 5.2. A Châtelet surface $X$ over a field $k$ is a smooth compactification of an affine surface defined in $\mathbb{A}_{k}^{3}$ by an equation of the form

$$
x^{2}-a y^{2}=P(z),
$$

where $P$ is a separable polynomial of degree 4 and $a \in k^{\times}$; see CTSSD87a, CTSSD87b.

To determine the Cox rings of $X$, one can start with the Cox ring $\bar{R}$ of $X_{\bar{k}}$ of identity type from [Der06, 6.4(iii)], descend it to a Cox ring $R$ of $X$ of type $\operatorname{id}_{\operatorname{Pic}\left(X_{\bar{k}}\right)}$ and compute a set of twists $R^{\sigma}$ representing all isomorphism classes of such Cox rings completely explicitly. Furthermore, one can compute a Cox ring of $X$ of type $\operatorname{Pic}(X) \hookrightarrow \operatorname{Pic}\left(X_{\bar{k}}\right)$.

If $a=-1$ and $P$ splits over $k$, this gives another explicit construction of the universal torsors and the split torsor described in [BBP12, §4], which are used in the proof of Manin's conjecture for such $X$ over $k=\mathbb{Q}$ [BBP12, Theorem 3.3]. See [Pie15, §2.4.3] for the details.

Also for other families of Châtelet surfaces, Cox rings and torsors of various types appear in proofs of Manin's conjecture. Based on our techniques, they are explicitly computed in [Des16, $\S 7.2-\S 7.3]$ and [Des18, $\S 4-\S 5]$.

Example 5.3. In the remainder of this section, we determine Cox rings of type $\operatorname{Pic}(X) \hookrightarrow \operatorname{Pic}\left(X_{\bar{k}}\right)$ for a smooth quintic del Pezzo surface $X$ that is a blow-up of the projective plane over a field $k$ in four conjugate points.

Proposition 5.4. Let $k$ be a field with separable closure $\bar{k}$. Let $\pi: X \rightarrow \mathbb{P}_{k}^{2}$ be a blow-up of four $\bar{k}$-points in general position that form one orbit under the $\mathrm{Gal}(\bar{k} / k)$ action. Let $Q_{1}, Q_{2} \in k\left[x_{0}, x_{1}, x_{2}\right]$ be non-proportional quadratic forms vanishing in the blown-up points.

Then a Cox ring of $X$ of type $\operatorname{Pic}(X) \hookrightarrow \operatorname{Pic}\left(X_{\bar{k}}\right)$ is given by the k-algebra

$$
R=k\left[\xi_{0}, \ldots, \xi_{5}\right] /\left(Q_{1}\left(\xi_{0}, \xi_{1}, \xi_{2}\right)-\xi_{3} \xi_{4}, Q_{2}\left(\xi_{0}, \xi_{1}, \xi_{2}\right)-\xi_{3} \xi_{5}\right),
$$

where $\operatorname{div}\left(\xi_{0}\right), \operatorname{div}\left(\xi_{1}\right), \operatorname{div}\left(\xi_{2}\right)$ are the total transforms of the three coordinate lines in $\mathbb{P}_{k}^{2}$, $\operatorname{div}\left(\xi_{3}\right)$ is the exceptional divisor of $\pi$, and $\operatorname{div}\left(\xi_{4}\right), \operatorname{div}\left(\xi_{5}\right)$ are the strict transforms of the conics defined by $Q_{1}, Q_{2}$, respectively.

Proof. Our starting point is the well-known description of Cox rings of identity type on $X_{\bar{k}}$ by the Plücker equations of $\operatorname{Gr}(2,5)$; see Pey98, Examples 3.3 .4 and 4.2.4], for example.

Let $E$ be the exceptional divisor of $\pi$. Let $\ell_{0}, \ldots, \ell_{4}$ be the basis of $\operatorname{Pic}\left(X_{\bar{k}}\right)$ where $\ell_{0}$ is the pullback of a hyperplane class in $\mathbb{P}_{k}^{2}$ and $\ell_{1}, \ldots, \ell_{4}$ are the classes of the four exceptional divisors of $\pi$ over $\bar{k}$. Then $\ell_{0}$ and $[E]=\ell_{1}+\ell_{2}+\ell_{3}+\ell_{4}$ are a basis of $\operatorname{Pic}(X)$. The generators $\xi_{i}$ with $D_{i}:=\operatorname{div}\left(\xi_{i}\right)$ of a Cox ring of type $\operatorname{Pic}(X) \hookrightarrow \operatorname{Pic}\left(X_{\bar{k}}\right)$ are computed by hand or using the MDS package HK15.

Let $\Lambda=\bigoplus_{i=0}^{5} \mathbb{Z} D_{i}$, let $\varphi: \Lambda \rightarrow \operatorname{Pic}\left(X_{\bar{k}}\right)$ send $D_{i}$ to $\left[D_{i}\right]$, and let $\Lambda_{0}=\operatorname{ker}(\varphi)$. Using the character $\chi: \Lambda_{0} \rightarrow k(X)^{\times}$defined by the conditions $\chi\left(D_{i}-D_{0}\right)=$ $\pi^{*}\left(x_{i} / x_{0}\right)$ for $i \in\{1,2\}$ and $\chi\left(D_{i}-\left(2 D_{0}-D_{3}\right)\right)=\pi^{*}\left(Q_{i-3}\left(x_{0}, x_{1}, x_{2}\right) / x_{0}^{2}\right)$ for $i \in\{4,5\}$, we find our two relations in degree $2 \ell_{0}$. The MDS package shows that there are no further relations.

In the situation of Proposition 5.4 let $-K$ be the anticanonical class on $X$. We observe that $R_{-K}$ is the $k$-vector space with basis $\left\{\xi_{i} \xi_{j}: i \in\{0,1,2\}, j \in\{4,5\}\right\}$. 
Since $-K$ is very ample, a torsor of type $\operatorname{Pic}(X) \hookrightarrow \operatorname{Pic}\left(X_{\bar{k}}\right)$ can be realized as $t: \operatorname{Spec} R \backslash V\left(R_{-K}\right) \rightarrow X$ by Corollary 4.3 .

Let $k$ be a number field and $\mathcal{O}_{k}$ its ring of integers. Let $\mathscr{Y}:=\operatorname{Spec} \mathscr{R} \backslash V\left(\mathscr{R}_{-K}\right)$ with

$$
\mathscr{R}:=\mathcal{O}_{k}\left[\xi_{0}, \ldots, \xi_{5}\right] /\left(Q_{1}\left(\xi_{0}, \xi_{1}, \xi_{2}\right)-\xi_{3} \xi_{4}, Q_{2}\left(\xi_{0}, \xi_{1}, \xi_{2}\right)-\xi_{3} \xi_{5}\right) .
$$

Applying [Pie15, Theorem 3.3] to ( $\left.\operatorname{Spec} \mathscr{R} ;\left\{\xi_{i} \xi_{j}: i \in\{0,1,2\}, j \in\{4,5\}\right\}\right)$, the $\mathcal{O}_{k}$-model $\mathscr{Y} \rightarrow \mathscr{X}$ of $t$ provided by Pie15, Construction 3.1] is a torsor under $\mathbb{G}_{m, \mathcal{O}_{k}}^{2}$.

For $k=\mathbb{Q}$, this gives is a $(4: 1)$-map from

$$
\mathscr{Y}(\mathbb{Z})=\left\{\left(\xi_{0}, \ldots, \xi_{5}\right) \in \mathbb{Z}^{6}: \begin{array}{l}
\operatorname{gcd}\left(\xi_{0}, \xi_{1}, \xi_{2}\right)=1, \operatorname{gcd}\left(\xi_{4}, \xi_{5}\right)=1 \\
Q_{1}\left(\xi_{0}, \xi_{1}, \xi_{2}\right)=\xi_{3} \xi_{4}, Q_{2}\left(\xi_{0}, \xi_{1}, \xi_{2}\right)=\xi_{3} \xi_{5}
\end{array}\right\}
$$

to $X(\mathbb{Q})$.

Remark 5.5 (D. Loughran). Consider the embedding $X \subset \mathbb{P}_{k}^{2} \times \mathbb{P}_{k}^{1}$ that we obtain by combining $\pi$ with the conic bundle structure $X \rightarrow \mathbb{P}_{k}^{1}$ corresponding to $Q_{1}, Q_{2}$. Then $X$ is defined by the equation $\xi_{4} \cdot Q_{2}\left(\xi_{0}, \xi_{1}, \xi_{2}\right)=\xi_{5} \cdot Q_{1}\left(\xi_{0}, \xi_{1}, \xi_{2}\right)$ in $\mathbb{P}_{k}^{2} \times$ $\mathbb{P}_{k}^{1}$ with coordinates $\left(\left(\xi_{0}: \xi_{1}: \xi_{2}\right),\left(\xi_{4}: \xi_{5}\right)\right)$. Over $k=\mathbb{Q}$, choosing primitive integral coordinates and defining $\xi_{3}=\operatorname{gcd}\left(Q_{1}\left(\xi_{0}, \xi_{1}, \xi_{2}\right), Q_{2}\left(\xi_{0}, \xi_{1}, \xi_{2}\right)\right)$ leads to our parameterization.

\section{REFERENCES}

[ADHL15] I. Arzhantsev, U. Derenthal, J. Hausen, and A. Laface. Cox rings, volume 144 of Cambridge Studies in Advanced Mathematics. Cambridge University Press, Cambridge, 2015.

[BB07] R. de la Bretèche and T. D. Browning. On Manin's conjecture for singular del Pezzo surfaces of degree four. II. Math. Proc. Cambridge Philos. Soc., 143(3):579-605, 2007.

[BBD07] R. de la Bretèche, T. D. Browning, and U. Derenthal. On Manin's conjecture for a certain singular cubic surface. Ann. Sci. École Norm. Sup. (4), 40(1):1-50, 2007.

[BBP12] R. de la Bretèche, T. Browning, and E. Peyre. On Manin's conjecture for a family of Châtelet surfaces. Ann. of Math. (2), 175(1):297-343, 2012.

[BBS14] V. Blomer, J. Brüdern, and P. Salberger. On a certain senary cubic form. Proc. Lond. Math. Soc. (3), 108(4):911-964, 2014.

[BH03] F. Berchtold and J. Hausen. Homogeneous coordinates for algebraic varieties. J. Algebra, 266(2):636-670, 2003.

[Bor63] M. Borelli. Divisorial varieties. Pacific J. Math., 13:375-388, 1963.

[BP04] V. V. Batyrev and O. N. Popov. The Cox ring of a del Pezzo surface. In Arithmetic of higher-dimensional algebraic varieties (Palo Alto, CA, 2002), volume 226 of Progr. Math., pages 85-103. Birkhäuser Boston, Boston, MA, 2004.

[Bre02] R. de la Bretèche. Nombre de points de hauteur bornée sur les surfaces de del Pezzo de degré 5. Duke Math. J., 113(3):421-464, 2002.

[BT13] R. de la Bretèche and G. Tenenbaum. Sur la conjecture de Manin pour certaines surfaces de Châtelet. J. Inst. Math. Jussieu, 12(4):759-819, 2013.

[BV17] N. Borne and A. Vistoli. Fundamental gerbes. arXiv:1610.07341v3, 2017.

[Cox95] D. A. Cox. The homogeneous coordinate ring of a toric variety. J. Algebraic Geom., 4(1):17-50, 1995.

[CT06] A.-M. Castravet and J. Tevelev. Hilbert's 14th problem and Cox rings. Compos. Math., 142(6):1479-1498, 2006.

[CT15] A.-M. Castravet and J. Tevelev. $\bar{M}_{0, n}$ is not a Mori dream space. Duke Math. J., 164(8):1641-1667, 2015.

[CTS77] J.-L. Colliot-Thélène and J.-J. Sansuc. Variétés de première descente attachées aux variétés rationnelles. C. R. Acad. Sci. Paris Sér. A-B, 284(16):A967-A970, 1977.

[CTS87] J.-L. Colliot-Thélène and J.-J. Sansuc. La descente sur les variétés rationnelles. II. Duke Math. J., 54(2):375-492, 1987.

[CTSSD87a] J.-L. Colliot-Thélène, J.-J. Sansuc, and P. Swinnerton-Dyer. Intersections of two quadrics and Châtelet surfaces. I. J. reine angew. Math., 373:37-107, 1987. 
[CTSSD87b] J.-L. Colliot-Thélène, J.-J. Sansuc, and P. Swinnerton-Dyer. Intersections of two quadrics and Châtelet surfaces. II. J. reine angew. Math., 374:72-168, 1987.

[Der06] U. Derenthal. Geometry of universal torsors (dissertation). Universität Göttingen, 2006.

[Der14] U. Derenthal. Singular del Pezzo surfaces whose universal torsors are hypersurfaces. Proc. Lond. Math. Soc. (3), 108(3):638-681, 2014.

[Des16] K. Destagnol. La conjecture de Manin pour certaines surfaces de Châtelet. Acta Arith., 174(1):31-97, 2016.

[Des18] K. Destagnol. Description de torseurs quasi-versels pour une famille de surfaces fibrées en coniques. arXiv:1711.01882v2, 2018.

[Dun16] A. Duncan. Twisted forms of toric varieties. Transform. Groups, 21(3):763-802, 2016.

[EKW04] E. J. Elizondo, K. Kurano, and K. Watanabe. The total coordinate ring of a normal projective variety. J. Algebra, 276(2):625-637, 2004.

[FMT89] J. Franke, Y. I. Manin, and Y. Tschinkel. Rational points of bounded height on Fano varieties. Invent. Math., 95(2):421-435, 1989.

[FP16] C. Frei and M. Pieropan. O-minimality on twisted universal torsors and Manin's conjecture over number fields. Ann. Sci. Éc. Norm. Supér. (4), 49(4):757-811, 2016.

[GOST15] Y. Gongyo, S. Okawa, A. Sannai, and S. Takagi. Characterization of varieties of Fano type via singularities of Cox rings. J. Algebraic Geom., 24(1):159-182, 2015.

[Gro03] A. Grothendieck. Revêtements étales et groupe fondamental (SGA 1). Documents Mathématiques (Paris), 3. Société Mathématique de France, Paris, 2003.

[Has09] B. Hassett. Rational surfaces over nonclosed fields. In Arithmetic geometry, volume 8 of Clay Math. Proc., pages 155-209. Amer. Math. Soc., Providence, RI, 2009.

[Hau08] J. Hausen. Cox rings and combinatorics. II. Mosc. Math. J., 8(4):711-757, 847, 2008.

[HK00] Y. Hu and S. Keel. Mori dream spaces and GIT. Michigan Math. J., 48:331-348, 2000.

[HK15] J. Hausen and S. Keicher. A software package for Mori dream spaces. LMS J. Comput. Math., 18(1):647-659, 2015.

[HT04] B. Hassett and Y. Tschinkel. Universal torsors and Cox rings. In Arithmetic of higher-dimensional algebraic varieties (Palo Alto, CA, 2002), volume 226 of Progr. Math., pages 149-173. Birkhäuser Boston, Boston, MA, 2004.

[KMRT98] M.-A. Knus, A. Merkurjev, M. Rost, and J.-P. Tignol. The book of involutions, volume 44 of American Mathematical Society Colloquium Publications. American Mathematical Society, Providence, RI, 1998.

[LU17] A. Laface and L. Ugaglia. Cox ring of the generic fiber. arXiv:1708.02797v1, 2017.

[MFK94] D. Mumford, J. Fogarty, and F. Kirwan. Geometric invariant theory, volume 34 of Ergebnisse der Mathematik und ihrer Grenzgebiete (2). Springer-Verlag, Berlin, third edition, 1994.

[Mil12] J. S. Milne. Algebraic geometry, version 5.22. Course Notes. http://www.jmilne.org/math/CourseNotes/ag.html 2012.

[Pey98] E. Peyre. Terme principal de la fonction zêta des hauteurs et torseurs universels. Astérisque, (251):259-298, 1998.

[Pie15] M. Pieropan. Torsors and generalized Cox rings for Manin's conjecture. PhD thesis, Leibniz Universität Hannover, 2015.

[Pie16] M. Pieropan. Imaginary quadratic points on toric varieties via universal torsors. Manuscripta Math., 150(3-4):415-439, 2016.

[Sal98] P. Salberger. Tamagawa measures on universal torsors and points of bounded height on Fano varieties. Astérisque, (251):91-258, 1998.

[Ser02] J.-P. Serre. Galois cohomology. Springer Monographs in Mathematics. SpringerVerlag, Berlin, english edition, 2002.

[Sko01] A. Skorobogatov. Torsors and rational points, volume 144 of Cambridge Tracts in Mathematics. Cambridge University Press, Cambridge, 2001.

[Sme17] A. Smeets. Insufficiency of the étale Brauer-Manin obstruction: towards a simply connected example. Amer. J. Math., 139(2):417-431, 2017.

[SS07] V. V. Serganova and A. N. Skorobogatov. Del Pezzo surfaces and representation theory. Algebra Number Theory, 1(4):393-419, 2007.

[TVAV11] D. Testa, A. Várilly-Alvarado, and M. Velasco. Big rational surfaces. Math. Ann., 351(1):95-107, 2011. 
Institut für Algebra, Zahlentheorie und Diskrete Mathematik, Leibniz Universität Hannover, Welfengarten 1, 30167 Hannover, Germany

E-mail address: derenthal@math.uni-hannover.de

EPFl SB MATH CAG, BÂt. MA, Station 8, 1015 Lausanne, Switzerland

E-mail address: marta.pieropan@epfl.ch 